\title{
Transport in a gravity dual with a varying gravitational coupling constant
}

\author{
Antonio M. García-García, Bruno Loureiro, and Aurelio Romero-Bermúdez \\ Cavendish Laboratory, University of Cambridge, \\ J.J. Thomson Avenue, Cambridge CB3 OHE, United Kingdom
}

(Received 30 June 2016; published 24 October 2016)

\begin{abstract}
We study asymptotically AdS Brans-Dicke (BD) backgrounds, where the Ricci tensor R is coupled to a scalar in the radial dimension, as effective models of metals with a varying coupling constant. We show that, for translationally invariant backgrounds, the regular part of the dc conductivity $\sigma_{Q}$ deviates from the universal result of Einstein-Maxwell-dilaton (EMD) models. However, the shear viscosity to entropy ratio saturates the Kovtun-Son-Starinets (KSS) bound. Similar results apply to more general f(R) gravity models. In four bulk dimensions we study momentum relaxation induced by gravitational and electromagnetic axion-dependent couplings. For sufficiently strong momentum dissipation induced by the former, a recently proposed bound on the dc conductivity $\sigma$ is violated for any finite electromagnetic axion coupling. Interestingly, in more than four bulk dimensions, the dc conductivity for strong momentum relaxation decreases with temperature in the low temperature limit. In line with other gravity backgrounds with momentum relaxation, the shear viscosity to entropy ratio is always lower than the KSS bound. The numerical computation of the optical conductivity reveals a linear growth with the frequency in the limit of low temperature, low frequency and large momentum relaxation. We have also shown that the module and argument of the optical conductivity for intermediate frequencies are not consistent with cuprates' experimental results, even assuming several channel of momentum relaxation.
\end{abstract}

DOI: 10.1103/PhysRevD.94.086007

\section{INTRODUCTION}

Einstein general relativity assumes that gravity is mediated by a tensor-two particle. Despite its immense conceptual and phenomenological success, generalizations [1] of general relativity, where gravity is also mediated by a scalar or a vector, have been intensively studied mostly for the potential interest in cosmology but also simply as toy models of new ideas in gravity. One of the most influential, though not the earliest [1], is the so-called Brans-Dicke gravity (BD) [2] that aimed to reconcile Mach's principle with general relativity. Gravity is also mediated by a scalar coupled linearly to the Ricci tensor. The action also has a kinetic term for the scalar so BD has two coupling constants. Physically this scalar can be understood as a gravitation constant $G$ that varies in time and space. General relativity is usually preferred as it predicts the same physics with fewer free parameters. Interestingly, after a conformal transformation, BD gravity maps onto Einstein gravity with a dilaton field. As a result of this mapping, explicit analytical solutions of the BD gravity equations of motion are known not only for Einstein gravity but also for asymptotically dS and AdS spaces even if the theory also contains massless photons modeled by the Maxwell tensor. For a certain region of parameters it is also possible [3] to map onto BD more general $f(R)$ models where the action is not linear in the Ricci tensor R.

In light of this rich phenomenology, we study asymptotically $\mathrm{AdS}$ BD backgrounds as effective duals of strongly coupled metals. Previous holographic studies $[4,5]$ involving BD backgrounds were restricted to thermodynamic properties only. By contrast here we focus on transport observables such as the optical, dc conductivity and shear viscosity in asymptotically AdS Brans-Dicke backgrounds. Our motivation is to explore the impact of the BD scalar running in the radial dimension, which acts as an effective gravitational constant, on the transport properties of holographic metals [6,7].

More specifically we address whether the universality of the shear viscosity [8] and the regular part of the dc conductivity [9-12], reported in translationally invariant Einstein-Maxwell-dilaton (EMD) [6,7] backgrounds with massless photons and no dilaton coupling to the Maxwell tensor, also holds in BD backgrounds. We have found that, while the universal shear viscosity ratio also holds in BD backgrounds, the finite part of the dc conductivity deviates from the universal result of EMD theories.

We also investigate momentum relaxation by gravitational axions, namely, axions coupled to the Ricci tensor, a simplified form of BD backgrounds where the scalar has no dynamics. Axions [13] together with massive gravitons, or simply a random chemical potential [14-19], break translational invariance which modifies substantially the conductivity and other transport properties. For weak momentum relaxation the conductivity is to a good extent described by Drude physics. For low temperatures or frequencies the conductivity is 
large, the so-called Drude peak, and decreases monotonously. It was observed, in all models studied, that no matter the strength of the momentum relaxation the conductivity of Einstein-Maxwell holographic metals was always above a certain bound which precludes a metal-insulator transition. In part based on this numerical evidence, the existence of a lower bound in the conductivity of more complicated holographic models was conjectured [20]. However, in two recent papers $[21,22]$ violations of this bound were reported in models where the axion is coupled to the Maxwell tensor, effectively screening charge. Here we show that gravitational axions, which do not screen charge, also lead to violations of the bound in the limit of strong, although still parametrically small with respect to the rank of the gauge group, axion gravitational coupling. For three space dimensions the dc conductivity decreases with $T$ for low temperatures even without any other source of momentum relaxation.

We also study the optical conductivity in BD backgrounds. The optical conductivity in EMD models with translational invariance in the limit of small frequencies and temperatures is controlled by the infrared (IR) geometry that for the Reissner-Nordström background is $\mathrm{AdS}_{2}$ leading to $\sigma \sim \omega^{2}$. The effect of momentum relaxation in the optical conductivity of EMD theories was investigated in [7] but it is not yet fully understood whether, for low frequencies and strong momentum relaxation, the conductivity scales as a power law faster than linear as in Mott insulators and many-body localized states [23].

By contrast, in a model in which momentum relaxation occurs by an oscillatory chemical potential, it was claimed [24] that the modulus of the optical conductivity for intermediate frequencies decays as a power law with an exponent equal to that observed in most cuprates. Here we find that, even assuming several channels of momentum dissipation, we cannot reproduce the modules and argument observed in cuprates. However, we have found that, for strong momentum dissipation and close to zero temperature, the optical conductivity increases linearly, not quadratically with the frequency, for both gravitational and electromagnetic axions. Finally, we have computed the ratio of the shear viscosity and the entropy density in BD holography with momentum relaxation. We have observed that, unlike the translationally invariant case, the ratio is temperature dependent. It decreases as the strength of momentum relaxation increases and it is always below the KSS bound. It can be made arbitrarily small for a finite amount of momentum relaxation.

The organization of the paper is as follows. In Sec. II we compute analytically the regular part of the dc conductivity and show that the shear viscosity to entropy ratio in translationally invariant $\mathrm{BD}$ backgrounds and other generalized theories of gravity is given by the KSS bound. In Sec. III we study the dc conductivity in BD-like backgrounds with momentum relaxation induced by coupling the axion and the Ricci tensor in two boundary space dimensions. In Sec. IV we address momentum relaxation by gravitational axions in higher space dimensions. In Sec. V we study the optical conductivity in BD backgrounds. We also compute the module and argument of the complex conductivity in order to compare with results in cuprates. In Sec. VI we compute the shear viscosity to entropy density ratio including different sources of momentum relaxation. We conclude with a list of interesting problems for further research and a short summary of the main results.

Next we introduce the BD action, the equations of motion and its analytical solution.

\section{DC CONDUCTIVITY IN TRANSLATIONALLY INVARIANT BD HOLOGRAPHY}

We start our analysis by introducing the BD action and the equations of motion (EOM). We then compute the conductivity for a general background ansatz and show how it is expressed in terms of thermodynamic quantities and the value of the scalar at the horizon. This is different from EMD models with no coupling between the dilaton and the Maxwell field where it only depends on thermodynamic quantities.

We then find that a calculation in the Einstein frame, resulting from a conformal transformation, leads to the same result. Finally, we discuss other modified gravity models that fall within the BD universality class.

\section{A. Brans-Dicke action and equations of motion}

The Brans-Dicke-Maxwell action in a $d+1$-dimensional manifold is given by

$S=\int_{\mathcal{M}} \mathrm{d}[d+1] \sqrt{-g}\left[\phi R-\frac{\xi}{\phi}(\nabla \phi)^{2}-V(\phi)-\frac{Y}{4} F^{2}\right]$.

Note that we are working in units where $2 \kappa^{2}=16 \pi G_{N}=1$ and we include a nontrivial coupling $Y(\phi)$ between the Brans-Dicke scalar $\phi$ and the Maxwell term, as well as a (for now arbitrary) scalar potential $V(\phi)$. In this model gravity is not only mediated by the massless symmetric rank two tensor $g$ but also by the real scalar field which has its own dynamics and a kinetic term parametrized by $\xi \geq 0 .{ }^{1}$ Intuitively the nonminimal coupling $\phi R$ can be interpreted as the running of Newton's constant " $G(x) \equiv G_{N} / \phi(x)$ " [3].

\footnotetext{
${ }^{1}$ The standard notation in the literature for the Brans-Dicke coupling is $\omega$. We refrain from this notation to avoid confusion with the frequency $\omega$ in the optical conductivity $\sigma(\omega)$.
} 
Variation of this action gives the following EOMs:

$$
\begin{aligned}
\phi\left(R_{a b}-\frac{1}{2} R g_{a b}\right)= & \frac{\xi}{\phi}\left(\nabla_{a} \phi \nabla_{b} \phi-\frac{1}{2}(\nabla \phi)^{2} g_{a b}\right) \\
& -\frac{1}{2} V(\phi)+\left(\nabla_{a} \nabla_{b} \phi-\square \phi g_{a b}\right) \\
& -\frac{Y}{2}\left(F_{a c} F_{b}^{c}+\frac{1}{4} F^{2} g_{a b}\right), \\
\partial_{a}\left(\sqrt{-g} Y(\phi) F^{a b}\right)= & 0, \\
\square \phi= & \frac{1}{2(d-1) \xi+2 d} \\
& \times\left((d-1) \phi V^{\prime}(\phi)-(d+1) V(\phi)\right. \\
& \left.-\frac{(d-3)}{4} F^{2}-\frac{Y^{\prime}(\phi)}{4} \phi F^{2}\right) .
\end{aligned}
$$

First, note that there is an extra term $\left(\nabla_{a} \nabla_{b} \phi-\square \phi g_{a b}\right)$ for the scalar in Einstein's equations. This term comes from the Palatini identity $\delta_{g} R=R_{a b} \delta g^{a b}-\nabla_{c}\left(\delta \Gamma_{a b}^{c}-g^{a c} \delta \Gamma_{c b}^{b}\right)$ which in Einstein gravity can be integrated to give a boundary term. Evaluating this term in normal coordinates and using Stoke's theorem yields the extra term previously mentioned. Second, terms on the left-hand side of the scalar equation come from the Ricci scalar factor that is solved by taking the trace of Einstein's equations.

An important observation is that for $Y=1$ and $d=3$ the Maxwell term in the scalar equation vanishes. This is a consequence of the fact that in $d=3$ the electromagnetic energy-momentum tensor is conformal and therefore traceless, and does not source the Ricci scalar. This invariance will play a crucial role later in our analysis.

\section{B. Regular part of the dc conductivity for a general ansatz}

We now present the computation of the conductivity at zero frequency in a generic static and spherically symmetric $\operatorname{AdS}_{d+1}$ black brane. As usual in translationally invariant theories, for vanishing frequency $\sigma(\omega \rightarrow 0) \rightarrow$ $\sigma_{Q}+K \delta(\omega)$, where we use the standard notation [11]: $K$ for the Drude weight and $\sigma_{Q}$ for the regular part of the dc conductivity. In this section we are only interested in the latter. We derive a general expression for $\sigma_{Q}$ that highlights the universality of our results. An explicit solution is worked out in Appendix A.

\section{Background and conserved charges}

Consider the following static and spherically symmetric ansatz for the field equations,

$$
\mathrm{d} s^{2}=-A(r) \mathrm{d} t^{2}+B(r) \mathrm{d} r^{2}+C(r) \delta_{i j} \mathrm{~d} x^{i} \mathrm{~d} x^{j}
$$

$$
\begin{gathered}
A=a_{t}(r) \mathrm{d} t, \\
\phi=\phi(r) .
\end{gathered}
$$

We assume that this chart is globally defined and describes an asymptotically $\mathrm{AdS}_{d+1}$ black hole. More precisely, we require that $A(r)=B(r)^{-1}=C(r)=r^{2}$ as $r \rightarrow \infty$ (asymptotic boundary) and that $A(r) \sim B(r)^{-1} \sim$ $4 \pi T\left(r-r_{0}\right)$ for $r_{0}>0$. Concerning the fields, we need to require that $Y(\infty)=1, \phi(\infty) \neq 0$ and $V(\phi) \sim 2 \Lambda \phi$ close to the boundary and that they are regular at the horizon $r=r_{0}$. Moreover we impose $a_{t}\left(r_{0}\right)=0$.

For this ansatz we use the existence of two radially conserved charges in order to simplify the computation of the conductivity. The simplest one is the charge density, which can be obtained by looking at the $(t)$ component of Maxwell's equations,

$$
\partial_{a}\left(\sqrt{-g} Y(\phi) F^{a t}\right)=\partial_{r}\left(\sqrt{-g} Y(\phi) g^{r r} g^{t t} a_{t}^{\prime}\right)=0 .
$$

Therefore the charge density

$$
\rho=\frac{Y C^{\frac{d-1}{2}}}{\sqrt{A B}} a_{t}^{\prime}
$$

is radially conserved. The second conserved quantity is related to the geometry. Consider the $(t t)$ and the $(x x)$ components of the Brans-Dicke equations with one raised index:

$$
\begin{aligned}
\phi\left(R_{t}^{t}-\frac{1}{2} R g_{t}^{t}\right)= & -\frac{Y}{2} F_{t r} F^{t r}+\left(-\frac{Y}{8} F^{2}+\frac{\xi}{2 \phi}(\nabla \phi)^{2}\right. \\
& \left.+\frac{1}{2} V-\square \phi\right) g_{t}^{t}+\nabla^{t} \nabla_{t} \phi, \\
\phi\left(R_{x}^{x}-\frac{1}{2} R g_{x}^{x}\right)= & \left(-\frac{Y}{8} F^{2}+\frac{\xi}{2 \phi}(\nabla \phi)^{2}+\frac{1}{2} V-\square \phi\right) g_{x}^{x} \\
& +\nabla^{x} \nabla_{x} \phi .
\end{aligned}
$$

Note that the assumption $\partial_{t} \phi=\partial_{x} \phi=0$ is crucial in this analysis. For our ansatz, $g_{t}^{t}=g_{x}^{x}=1$ and therefore we subtract the above expression to give

$$
\phi\left(R_{t}^{t}-R_{x}^{x}\right)=-\frac{Y}{2} F_{r t} F^{r t}+\nabla^{t} \nabla_{t} \phi-\nabla^{x} \nabla_{x} \phi .
$$

In order to write this as a total derivative, we will make use of the following identities,

$$
\begin{aligned}
& \sqrt{-g} R_{t}^{t}=-\frac{1}{2} \partial_{r}\left(\frac{1}{\sqrt{A B}} C^{\frac{d-1}{2}} A^{\prime}\right), \\
& \sqrt{-g} R_{x}^{x}=-\frac{1}{2} \partial_{r}\left(\sqrt{\frac{A}{B}} C^{\frac{d-3}{2}} C^{\prime}\right),
\end{aligned}
$$


which are subtracted to give

$$
\sqrt{-g}\left(R_{t}^{t}-R_{x}^{x}\right)=-\frac{1}{2} \partial_{r}\left(\frac{1}{\sqrt{A B}} C^{\frac{d+1}{2}}\left(\frac{A}{C}\right)^{\prime}\right) .
$$

Regarding the right-hand side, we have

$$
\begin{aligned}
\nabla^{t} \nabla_{t} \phi-\nabla^{x} \nabla_{x} \phi & =g^{t t} \nabla_{t} \nabla_{t} \phi-g^{x x} \nabla_{x} \nabla_{x} \phi \\
& =-\left(g^{t t} \Gamma_{t t}^{r}-g^{x x} \Gamma_{x x}^{r}\right) \phi^{\prime} \\
& =\frac{1}{2 B}\left(\frac{A^{\prime}}{A}-\frac{C^{\prime}}{C}\right) \phi^{\prime},
\end{aligned}
$$

and therefore

$$
\sqrt{-g}\left(\nabla^{t} \nabla_{t} \phi-\nabla^{x} \nabla_{x} \phi\right)=\frac{C^{\frac{d+1}{2}}}{2 \sqrt{A B}}\left(\frac{A}{C}\right)^{\prime} \phi^{\prime}
$$

Taking into account that $\sqrt{-g} Y F^{r t}=\rho$, we finally rewrite Eq. (6) as

$$
\phi \partial_{r}\left(\frac{1}{\sqrt{A B}} C^{\frac{d+1}{2}}\left(\frac{A}{C}\right)^{\prime}\right)=\rho a_{t}^{\prime}-\frac{C^{\frac{d+1}{2}}}{\sqrt{A B}}\left(\frac{A}{C}\right)^{\prime} \phi^{\prime},
$$

which is expressed as a total derivative,

$$
\partial_{r}\left(\frac{\phi}{\sqrt{A B}} C^{\frac{d+1}{2}}\left(\frac{A}{C}\right)^{\prime}-\rho a_{t}\right)=0 .
$$

In previous works, this conserved charge was related to thermodynamical quantities [9-11]. Indeed, by integrating the conserved charge, evaluating it at the horizon and using the boundary condition $a_{t}\left(r_{0}\right)=0$ we get

$$
\left.\frac{\phi}{\sqrt{A B}} C^{\frac{d+1}{2}}\left(\frac{A}{C}\right)^{\prime}\right|_{r=r_{0}}=\frac{\phi\left(r_{0}\right)}{\sqrt{A B}} C\left(r_{0}\right)^{\frac{d-1}{2}} A^{\prime}\left(r_{0}\right)=s T
$$

which is exactly the same result obtained previously. Note that we use the fact that the entropy in BD theory satisfies

$$
S=\frac{1}{4} \int_{r=r_{0}} \mathrm{~d}[d] x \phi \sqrt{-g}
$$

instead of the standard area law. This is another simple manifestation of the idea that the strength of gravity in BD backgrounds is not constant, as in Einstein gravity, but governed by the scalar " $G / \phi$ " [3]. ${ }^{2}$ Surprisingly, the scalar field fits nicely to produce the same thermodynamic result.

\footnotetext{
${ }^{2}$ Note that in standard units the area law is $S=\frac{1}{4 G} \int_{r=r_{0}} \mathrm{~d}[d] x \sqrt{-g}$.
}

For a general $r$, we then have

$$
s T=\frac{\phi}{\sqrt{A B}} C^{\frac{d+1}{2}}\left(\frac{A}{C}\right)^{\prime}-\rho a_{t} .
$$

Note that in particular $a_{t}(\infty)=\mu$. Therefore using the Smarr relation $s T+\mu \rho=\epsilon+P$ and the above expression evaluated at the boundary $r \rightarrow \infty$ we get

$$
\epsilon+P=\left.\frac{\phi}{\sqrt{A B}} C^{\frac{d+1}{2}}\left(\frac{A}{C}\right)^{\prime}\right|_{r=\infty} .
$$

\section{Fluctuations}

In order to compute conductivities, we need to study fluctuations around the background solutions. It is sufficient to consider the following set of consistent fluctuations:

$$
\mathrm{d} s^{2} \rightarrow \mathrm{d} s^{2}+2 h_{t x}(t, r) \mathrm{d} t \mathrm{~d} x, \quad A \rightarrow A+a_{x}(t, r) \mathrm{d} x .
$$

The EOM for $a_{x}$ is obtained by linearizing the $(x)$ component of Maxwell's equations,

$$
\begin{aligned}
& \partial_{a}\left(\sqrt{-g} Y g^{a c} g^{x d} F_{c d}\right) \\
& =\partial_{r}\left(\sqrt{-g} Y g^{r r} g^{x x} \partial_{r} a_{x}\right)-\partial_{r}\left(\sqrt{-g} Y g^{r r} g^{x t} a_{t}^{\prime}\right) \\
& \quad+\partial_{t}\left(\sqrt{-g} Y g^{t t} g^{x x} \partial_{t} a_{x}\right) \\
& =\partial_{r}\left(\sqrt{\frac{A}{B}} C^{\frac{d-3}{2}} Y \partial_{r} a_{x}\right)-\sqrt{\frac{B}{A}} C^{\frac{d-3}{2}} Y \partial_{t}^{2} a_{x}+\rho \partial_{r}\left(g^{x x} h_{t x}\right)=0
\end{aligned}
$$

To eliminate the $h_{t x}$ term in the above expression we look at the constraint equation given by the linearized $(r x)$ Brans-Dicke equation,

$$
\partial_{r}\left(g^{x x} h_{t x}\right)=-\frac{g^{x x}}{\phi} Y a_{t}^{\prime} a_{x}
$$

Inserting this in the above expression and solving for $a_{t}^{\prime}$ in the function of the charge density we get

$$
\frac{\phi C^{\frac{d+1}{2}}}{\sqrt{A B}} \partial_{r}\left(\sqrt{\frac{A}{B}} C^{\frac{d-3}{2}} Y \partial_{r} a_{x}\right)-\sqrt{\frac{B}{A}} C^{\frac{d-3}{2}} Y \partial_{t}^{2} a_{x}-\rho^{2} a_{x}=0 .
$$

We now use the conserved charge (8) to rewrite the above equation as

$$
\partial_{r}\left(\frac{C^{d-1} Y \phi}{B}\left(\frac{A}{C}\right)^{\prime} \partial_{r} a_{x}-\frac{A}{C} \rho^{2} a_{x}\right)-\sqrt{\frac{B}{A}} C^{\frac{d-3}{2}} Y \partial_{t}^{2} a_{x}=0 .
$$

As long as we are only interested in the regular part of the dc conductivity, we can set $\partial_{t}^{2} a_{x}=0$. The resulting equation is easily integrated to give 


$$
\frac{C^{d-1} Y \phi}{B}\left(\frac{A}{C}\right)^{\prime} \partial_{r} a_{x}-\frac{A}{C} \rho^{2} a_{x}=\text { constant. }
$$

Black brane boundary conditions set $A\left(r_{0}\right) \sim 1 / B\left(r_{0}\right)=0$. Regularity of the fields at the horizon fixes the constant above to zero. Moreover, without loss of generality we set $\lim _{r \rightarrow \infty} \phi(r) \equiv 1$ at the boundary. With this information the equation for the fluctuation is easily integrated:

$$
\begin{aligned}
a_{x}^{(0)}(r) & =\exp \left\{-\int_{r}^{\infty} \frac{A B \rho^{2}}{Y \phi(A / C)^{\prime} C^{d-2}} \mathrm{~d} r\right\} \\
& =\exp \left\{-\int_{r}^{\infty} \frac{Y\left(a_{t}^{\prime}\right)^{2}}{\phi C(A / C)^{\prime}} \mathrm{d} r\right\},
\end{aligned}
$$

where $a_{x}^{(0)}(r)$ is the independent solution of the equation that tends to 1 at the boundary and determines the regular part of the conductivity. ${ }^{3}$ We can use the charges (5) and (9) to perform the integral explicitly:

$$
\begin{aligned}
\int_{r}^{\infty} \frac{Y\left(a_{t}^{\prime}\right)^{2}}{\phi C(A / C)^{\prime}} \mathrm{d} r & =\int_{r}^{\infty} \frac{a_{t}^{\prime}}{a_{t}+s T / \rho} \mathrm{d} r \\
& =\left.\log \left(a_{t}(r) \rho+s T\right)\right|_{r} ^{\infty} \\
& =\log \frac{\epsilon+P}{a_{t}(r) \rho+s T},
\end{aligned}
$$

where we have used the Smarr relation. This implies that

$$
a_{x}^{(0)}(r)=\frac{a_{t}(r) \rho+s T}{\epsilon+P},
$$

and in particular

$$
a_{x}^{(0)}\left(r_{0}\right)=\frac{s T}{\epsilon+P}
$$

where we use the boundary condition $a_{t}\left(r_{0}\right)=0$. Finally, the regular part of the dc conductivity is given by

$$
\begin{aligned}
\sigma_{Q} & =Y\left(r_{0}\right) C\left(r_{0}\right)^{\frac{d-3}{2}}\left(a_{x}^{(0)}\left(r_{0}\right)\right)^{2} \\
& =Y\left(r_{0}\right) C\left(r_{0}\right)^{\frac{d-3}{2}}\left(\frac{s T}{\epsilon+P}\right)^{2} .
\end{aligned}
$$

This result is the same as the one obtained in EMD models [9-11]. It is interesting to observe that in these works $T_{t}^{t}=T_{x}^{x}$ is given as a necessary condition for the universality of $\sigma_{Q}$. Here this condition is clearly violated, but we can still write the equation for the fluctuation as a total derivative. However, taking into account the modified area law for the entropy density,

\footnotetext{
${ }^{3}$ The second solution can be obtained using the Wronskian. For further details see [11].
}

$$
\sigma_{Q}=\frac{Y\left(r_{0}\right)}{\phi\left(r_{0}\right)^{\frac{d-3}{d-1}}}\left(\frac{s}{4 \pi}\right)^{\frac{d-3}{d-1}}\left(\frac{s T}{\epsilon+P}\right)^{2}
$$

we highlight the explicit dependence of $\sigma_{Q}$ on the BD field $\phi$. For $d>3$, we thus expect the scalar field to renormalize the universal contribution to the conductivity. To understand better the possible effects of $\phi$, we need to evaluate its behavior at the horizon, in particular, the low and high temperature scaling of $\phi\left(r_{0}\right)$.

In contrast with the previous discussion, those questions do not have a universal answer. It depends on the particular solution for the background. In the next section, we will see that the BD model can be formally mapped onto an EMD model by a conformal transformation. Explicit solutions for the latter have been widely studied in both the context of gravity $[25,26]$ and of holography $[6,27]$ (and references therein). As an illustration of this method we study in Appendix A a particular solution, and show explicitly how it is mapped to BD.

A simple numeric fit for the solution (A1) suggests two scaling regimes. For low temperature, $\phi(T) \sim a$ tends, for a fixed charged density, to a temperature-independent constant $0<a<1$. This indicates that close to extremality the temperature scaling of $\sigma_{Q}$ coincides with the EMD result, although up to a numerical prefactor $a$. For high temperatures, we find that $\phi(T) \sim T^{-\delta}$ for $\delta \geq 0$, suggesting that $\phi \rightarrow 0$ asymptotically at the horizon for sufficiently high temperatures. Here $\delta$ is a function of both the Brans-Dicke parameter $\xi$ and the dimensionality $d$, and monotonically decreases with $\xi \geq 0$ for fixed $d$. For example, for $d=4$ and $\xi=0$, we have $\delta \approx 0.4$, while $\delta \approx 0.22$ for $\xi=1$. In particular we have $\delta \rightarrow 0$ as $\xi \rightarrow \infty$ for any dimension $d>2$.

One can interpret this behavior in a heuristic way. First note that Newton's constant $G$ is related to the string coupling constant $g_{s} \sim G$. If we naively interpret the BD coupling $G / \phi$ as a dynamical Newton's constant, the flow of $\phi$ can be interpreted as a flow from weaker $(\phi \gg 1)$ to stronger $(\phi \ll 1)$ coupling. More specifically, for the background solution of Appendix A $\phi \in\left[0, \phi\left(r_{0}\right)\right]$ with $0<\phi\left(r_{0}\right) \leq 1$. Therefore the running of $\phi$ from the boundary to the horizon corresponds in the dual field theory to a flow from weaker to stronger coupling. For the purpose of the conductivity, this running has the effect of increasing $\sigma_{Q}$ by a factor $\phi\left(r_{0}\right)^{-\frac{d-3}{d-1}}$. Although tempting, one needs to be cautious with this heuristic interpretation. In the saddle point approximation, exact only in the $N \rightarrow \infty$ limit, we always have $g_{s} \ll 1$ and $\lambda=g_{s} N \gg 1$. Thus this interpretation should not be taken seriously in the limit of fixed large $N$ and $\phi\left(r_{0}\right) \rightarrow 0$, where the saddle point is clearly not valid. ${ }^{4}$

\footnotetext{
${ }^{4}$ For this reason we employ the term "weaker" instead of "weak."
} 


\section{Conformal transformations and universality}

The explicit result for the regular part of the dc conductivity (10) is also expected from a well-known trick broadly used in the Brans-Dicke literature that we now discuss (see for example [28] and references within).

Consider the following conformal mapping of the metric $g$,

$$
\bar{g}=\phi^{\frac{2}{d-1}} g .
$$

Taking into account the transformation in the volume element and in the Ricci scalar, the action reads

$$
\bar{S}=\int_{\mathcal{M}} \mathrm{d}[d+1] \sqrt{-g}\left[\bar{R}-\frac{4}{d-1}(\bar{\nabla} \bar{\phi})^{2}-\bar{V}(\bar{\phi})-\frac{\bar{Y}(\bar{\phi})}{4} \bar{F}^{2}\right],
$$

where we have defined

$$
\begin{array}{ll}
\alpha=\frac{d-3}{2 \sqrt{(d-1) \xi+d}} & \bar{\phi}=\frac{d-3}{4 \alpha} \log \phi \\
\bar{V}(\bar{\phi})=\phi^{-\frac{d+1}{d-1} V(\phi)} & \bar{Y}(\bar{\phi})=Y(\bar{\phi}) e^{-\frac{4 \alpha \bar{\phi}}{d-1}}
\end{array}
$$

and all bar.- quantities are computed with respect to the metric $\bar{g}$. Note that for $d=3$ the Maxwell coupling is not affected by the conformal mapping. This is a consequence of the fact that electromagnetism is conformal in $d=3$. It is also useful to note that $\bar{\phi}$ is well defined for $d=3$ since $\alpha$ has a factor $d-3$ as well.

This is nothing but the well-known Einstein-Maxwelldilaton action. This action has been widely studied in the context of string theory and effective holographic models [5-7,29]. This map provides a useful way of constructing solutions to BD gravity, since solutions of EMD theory are well known. A particularly explicit solution that illustrates this point is given in Appendix A. More interestingly, it is known that the regular part of the dc conductivity in such models takes the (almost) universal form

$$
\bar{\sigma}_{Q}=\bar{Y}\left(r_{0}\right)\left(\frac{\bar{s}}{4 \pi}\right)^{\frac{d-3}{d-1}}\left(\frac{\bar{s} \bar{T}}{\bar{\epsilon}+\bar{P}}\right)^{2} .
$$

It is not hard to check that the thermodynamic quantities $(\bar{s}, \bar{T}, \bar{\epsilon}, \bar{P})$ are invariant under the conformal mapping. ${ }^{5}$ The only part of $\bar{\sigma}_{Q}$ that is not invariant is the nonuniversal charge coupling $\bar{Y}$, which transforms as $\bar{Y}(\bar{\phi})=\phi^{-\frac{d-3}{d-1} Y} Y(\phi)$ and gives the explicit result computed in the previous section.

This raises the interesting question of whether there are other theories of modified gravity that can be cast as an

\footnotetext{
${ }^{5}$ This is essentially a consequence of the regularity of $\phi$ at the horizon.
}

EMD theory in the Einstein frame, and if so, which those theories are. Indeed, this question has been much discussed in the gravity literature [30-32]. There has been a controversial debate on whether theories that are related by field redefinition or conformal transformation describe gravitationally ${ }^{6}$ the same theory or not [33]. A full discussion of those intricate questions is beyond the scope of the paper. Here we limit our discussion to the fact that conformal transformations are a convenient tool to study dynamically equivalent theories.

A well-known class of theories that can be mapped into BD are $f(R)$ theories of gravity, defined through the action

$$
S=\int_{\mathcal{M}} \mathrm{d}[d+1] x \sqrt{-g} f(R)+S_{\text {matter }},
$$

where $f(R)$ is a generic function of the Ricci scalar $R$ and $S_{\text {matter }}$ includes any other fields coupled to the metric, but not to $R$. One can introduce an auxiliary field $\chi$ to rewrite the above equation as

$$
S=\int_{\mathcal{M}} \mathrm{d}[d+1] x \sqrt{-g}\left[f(\chi)+f^{\prime}(\chi)(R-\chi)\right]+S_{\text {matter }} .
$$

Variation with respect to $\chi$ gives $f^{\prime \prime}(\chi)(R-\chi)=0$, so as long as $f^{\prime \prime}(\chi) \neq 0$ this constraint imposes $\chi=R$ and we recover the initial action. Note that this is also a sufficient condition for $f(R)$ to be invertible. Now defining $\phi=f^{\prime}(\chi)$ and $V(\phi)=\chi(\phi) \phi-f(\chi(\phi))$ we can write

$$
S=\int_{\mathcal{M}} \mathrm{d}[d+1] x \sqrt{-g}(\phi R-V(\phi))+S_{\text {matter }},
$$

which is precisely a BD action with $\xi=0$ and potential $V(\phi)$. This procedure is nothing but the Legendre transform of the action with respect to the conjugate field $\phi$. Taking $S_{\text {matter }}=-\frac{1}{4} \int Y(r) F^{2}$, we proceed with the computation of the regular part of the conductivity as before to get

$$
\sigma_{Q}=\frac{Y\left(r_{0}\right)}{f^{\prime}\left(R\left(r_{0}\right)\right)^{\frac{d-3}{d-1}}}\left(\frac{s}{4 \pi}\right)^{\frac{d-3}{d-1}}\left(\frac{s T}{\epsilon+P}\right)^{2} .
$$

This illustrates how a combination of a conformal transformation and a Legendre transform can be used to considerably simplify calculations. Indeed, this procedure is much more general, and can be applied to other theories such as Palatini gravity or $f(\phi)$ couplings to the Ricci [33]. An interesting example of the latter is a conformal coupling $f(\phi)=1+\frac{1}{6} \phi^{2}$ that appears naturally in one-loop diagrams of string theory [34].

\footnotetext{
${ }^{6} \mathrm{By}$ gravitationally we mean the geodesics, conservation of the energy-momentum tensor, energy conditions, etc.
} 
It is tempting to apply this construction to other theories of gravity such as Gauss-Bonnet, which in holography effectively correspond to leading $1 / N$ corrections in the dual field theory. However, Gauss-Bonnet theories contain terms such as $R_{a b} R^{a b}$ which introduce further nonlinearity and thus make the Legendre transformation difficult [32]. Therefore Gauss-Bonnet gravity does not fall under BD universality.

A natural question to ask is what happens with other transport coefficients. For example, both the shear viscosity and the entropy contain the same power of $G$, and therefore $\eta / s$ does not depend on $G$. As a consequence, we expect $\eta / s=1 / 4 \pi$ in BD holography to saturate the KSS bound. This is just a particular example of a general result that any theory related to standard gravity via a conformal transformation indeed saturates the KSS bound [35,36]. However, quantities such as the entanglement entropy should be sensitive to $\phi$ in the expected way $\left(G \rightarrow G \phi^{-1}\right)$. Indeed this was explicitly calculated in the context of $f(R)$ theories, and agrees with our discussion since $\phi=f^{\prime}(R)$ [37-39].

This discussion applies only to theories with no momentum relaxation. In the rest of the paper we focus on the description of transport in BD holography with momentum dissipation.

\section{MOMENTUM RELAXATION AND DC CONDUCTIVITY IN BD HOLOGRAPHY}

We now study the effect of momentum relaxation in the transport properties of the field theory dual of BD gravity. We consider the linear coupling to the Ricci scalar to be a function of the gradient of axion fields that explicitly break diffeomorphism invariance in the boundary spacelike coordinates:

$$
\begin{aligned}
S= & \int \mathrm{d}[d+1] x \sqrt{-g}[Z(\operatorname{Tr} X) R-2 \Lambda \\
& \left.-V(\operatorname{Tr} X)-\frac{Y(\operatorname{Tr} X)}{4} F^{2}\right],
\end{aligned}
$$

where $\operatorname{Tr} X=\frac{1}{d-1} \sum_{I} \nabla_{\mu} X^{I} \nabla^{\mu} X^{I}$ and $X^{I}=\alpha x^{I}$. We use the metric ansatz of Eq. (2) with $A=g, B=1 / g$ and $C=r^{2} c$ :

$$
\begin{aligned}
\mathrm{d} s^{2} & =-g(r) \mathrm{d} t^{2}+\frac{\mathrm{d} r^{2}}{g(r)}+r^{2} c(r) \delta_{i j} d x^{i} d x^{j}, \\
i & =1, \ldots, d-1, \quad r \in\left[r_{0}, \infty\right) .
\end{aligned}
$$

Assuming that $g \rightarrow r^{2}$ and $c \rightarrow 1$ for large $r$ and $g$ has a (double) single zero at (zero) finite temperature, which defines the horizon, we follow the procedure devised by Donos and Gauntlett [40] to compute the dc conductivity from the solution of the EOMs at the horizon.
We add a perturbation in $A_{x}$ linear in time, while the axion and metric perturbations are independent of time,

$$
\begin{aligned}
A & \rightarrow A+\left(a_{x}(r)-E t\right) \mathrm{d} x, \quad X^{x} \rightarrow X^{x}+\chi(r), \\
\mathrm{d} s^{2} & \rightarrow \mathrm{d} s^{2}+2 r^{2} h_{t x}(r) \mathrm{d} t \mathrm{~d} x+2 r^{2} h_{r x}(r) \mathrm{d} r \mathrm{~d} x .
\end{aligned}
$$

Maxwell's equation for $a_{x}$ is

$$
\partial_{r}\left[Y \sqrt{-g} g^{r r}\left(g^{t x} F_{r t}+g^{x x} F_{r x}\right)\right]=0,
$$

which leads to the radially conserved quantity:

$$
J=-Y r^{d-3} c^{\frac{d-3}{2}} g a_{x}^{\prime}-h_{t x} \frac{\rho}{c} .
$$

This conserved quantity is evaluated at the horizon where $h_{t x}$ and $a_{x}$ are obtained as we discuss below.

The perturbation on the gauge field close to the horizon is obtained from Eq. (16) by choosing $J$ such that $a_{x}$ is ingoing in the horizon:

$$
a_{x}^{\prime} \sim-\frac{E}{g} \Rightarrow a_{x} \sim-E v,
$$

where $v$ is the ingoing Eddington-Finkelstein coordinate $v=t+r_{*}$, given in terms of the tortoise coordinate $d r_{*}=\frac{d r}{g}$.

Equation (17) gives the first term inside the parentheses of Eq. (16). To obtain the second term, we combine the $(x t)$ and $(x x)$ Einstein's equations. Since we will evaluate them at the horizon, we will only write down explicitly the nonzero terms after taking the limit $r \rightarrow r_{0}$.

For clarity we write down Einstein's equations only for $d=3$ :

$$
\begin{aligned}
Z G_{a b}= & \frac{1}{2} T_{a b}+\frac{1}{2} \mathcal{Z}_{a b}, \\
T_{a b}= & Y\left(F_{a}^{c} F_{b c}-\frac{1}{4} g_{a b} F^{2}\right)-(2 \Lambda+V) g_{a b} \\
& +\sum_{I} \nabla_{a} X^{I} \nabla_{b} X^{I}\left(-\dot{V}-\frac{\dot{Y}}{4} F^{2}+\dot{Z} R\right), \\
\mathcal{Z}_{a b}= & 2\left(\nabla_{a} \nabla_{b}-g_{a b} \nabla_{c} \nabla^{c}\right) Z,
\end{aligned}
$$

where the dot derivative stands for derivative with respect to $\operatorname{Tr} X=\frac{1}{2} g^{a b} \sum_{I} \partial_{a} X^{I} \partial_{b} X^{I}$ and $R$ is the Ricci scalar. ${ }^{7}$

\footnotetext{
${ }^{7}$ The full dynamical stability of the model Eq. (13) is beyond the scope of the paper. We thank Oriol Pujolàs and Matteo Baggioli for pointing out the occurrence of third order time derivatives. These occur beyond the linear analysis and have the potential to further restrict the parameters for which the model is stable.
} 
The $(t x)$ Einstein's equation is

$$
\begin{aligned}
\mathcal{O}\left(h_{t x}{ }^{\prime}, h_{t x}{ }^{\prime \prime}\right)+\frac{r^{2}}{2} Z h_{t x} r^{2}\left[g^{\prime \prime}+g^{\prime}\left(\frac{2}{c}+\frac{c^{\prime}}{c}\right)+\cdots\right] \\
=\frac{r^{2}}{4} h_{t x}\left(-4 \Lambda-2 V+Y a_{t}^{\prime 2}\right)+\frac{1}{2} g Y a_{t} a_{x}^{\prime} \\
\quad+\frac{1}{2} r^{2} g \dot{Z} \operatorname{Tr} X^{\prime} h_{t x}{ }^{\prime}-\frac{r h_{t x}}{2 c}\left[2 r c g^{\prime} \dot{Z} \operatorname{Tr} X^{\prime}+\cdots\right],
\end{aligned}
$$

where the dots and the terms $\mathcal{O}\left(h_{t x}{ }^{\prime}, h_{t x}{ }^{\prime \prime}\right)$ are zero at the horizon, the prime derivative is with respect to $r$ and $\operatorname{Tr} X^{\prime}=\partial_{r} \operatorname{Tr} X$. The first two terms on the right-hand side come from $T_{a b}$ and the last term from $\mathcal{Z}_{a b}$. In order to simplify Eq. (19) we eliminate $c^{\prime \prime}(r)$ from the $(t t)$ Einstein's equation and substitute it into the $(x x)$ Einstein's equation. The result is given in Eq. (20) by specifying $G_{x x}$, $T_{x x}$ and $\mathcal{Z}_{x x}$ separately,

$$
\begin{aligned}
Z G_{x x}= & \frac{r^{2} c}{2} Z g^{\prime \prime}+\frac{r^{2} Z}{4}\left(\frac{c^{\prime}}{c}+\frac{2}{r}\right) g^{\prime} \\
& +\frac{r^{4} Z}{8} \frac{4 \Lambda+2 V+Y a_{t}^{\prime 2}}{k^{2} \dot{Z}-r^{2} c Z}+\cdots, \\
\mathcal{Z}_{x x}= & \frac{\alpha^{2} \dot{Z} g^{\prime}}{2}\left(\frac{c^{\prime}}{c}+\frac{2}{r}\right)+\frac{\alpha^{2} r^{2} c \dot{Z}}{4} \frac{4 \Lambda+2 V+Y a_{t}^{\prime 2}}{\alpha^{2} \dot{Z}-r^{2} c Z}+\cdots, \\
\frac{1}{2} T_{x x}= & \frac{\alpha^{2} \dot{Z}}{2} g^{\prime \prime}+\frac{\alpha^{2} \dot{Z}}{2}\left(\frac{c^{\prime}}{c}+\frac{2}{r}\right) g^{\prime} \\
& +\frac{r^{2} Z}{4} \frac{r^{2} c\left(4 \Lambda+2 V-Y a_{t}^{\prime 2}\right)+\alpha^{2}\left(-2 \dot{V}+\dot{Y} a_{t}^{\prime 2}\right)}{\alpha^{2} \dot{Z}-r^{2} c Z} \\
& +\frac{\dot{Z}}{4} \frac{2 \alpha^{2} \dot{V}+\left(2 \alpha^{2} r^{2} c Y-\alpha^{4} \dot{Y}\right) a_{t}^{\prime 2}}{\alpha^{2} \dot{Z}-r^{2} c Z}+\cdots,
\end{aligned}
$$

where the dots vanish at the horizon. Combining Eqs. (20) and (19) allows us to eliminate $h_{t x}$. Its value at the horizon $r=r_{0}$ is used to calculate the dc conductivity from $\operatorname{Re}\left(\sigma_{\mathrm{dc}}\right)=J / E$. Before we do so, we define the expansions of the metric functions $g$ and $c$ close to the horizon as

$$
\begin{aligned}
& g \sim g_{1}\left(1-\frac{r_{0}}{r}\right)+\cdots, \\
& c \sim c_{0}+c_{1}\left(1-\frac{r_{0}}{r}\right)+\cdots .
\end{aligned}
$$

Restoring arbitrary bulk dimensionality $d+1$, the temperature is

$$
T=\frac{1}{4 \pi} \frac{c_{0} r_{0}}{2 c_{0}+c_{1}} \frac{4 \Lambda+2 V\left(r_{0}\right)+Y\left(r_{0}\right) a_{t}^{\prime}\left(r_{0}\right)^{2}}{\frac{2 \alpha^{2} \dot{Z}\left(r_{0}\right)}{r_{0}^{2} c_{0}}-(d-1) Z\left(r_{0}\right)},
$$

where we have used $\operatorname{Tr} X=\frac{\alpha^{2}}{r^{2} c}$ to simplify the denominator. The dc conductivity, obtained from Eqs. (16) and (17), and the value of $h_{t x}$ at the horizon (calculated as indicated previously), is given by the following compact expression,

$$
\begin{aligned}
\operatorname{Re}\left(\sigma_{\mathrm{dc}}\right) & =Y_{0} r_{0}^{d-3} c_{0}^{\frac{d-3}{2}}+\frac{\rho^{2}}{m_{\mathrm{eff}}^{2}}, \\
m_{\mathrm{eff}}^{2} & =2 c_{0}^{\frac{d-1}{2}} r_{0}^{d-1}\left(T \mathcal{A}+Z_{0}^{2} \mathcal{B}+\dot{Z}_{0} \mathcal{C}\right)
\end{aligned}
$$

where $T$ is the temperature from Eq. (22) and $\mathcal{A}, \mathcal{B}$ and $\mathcal{C}$ are given in Eq. (24). The term $a_{t}^{\prime}=\frac{\rho}{Y(r) r^{d-1} c(r)^{\frac{d-1}{2}}}$ is also evaluated at the horizon. The subscripts 0 in Eqs. (23) and (24) indicate that the variable is evaluated at the horizon.

Equation (23) suggests that even at zero temperature the conductivity receives a correction given by the $\dot{Z}_{0}^{2} \mathcal{C}$ term. We note that although this is a fully analytical expression for the conductivity the metric at the horizon may only be computed numerically:

$$
\begin{aligned}
\mathcal{A}= & \frac{4 \pi\left(2 c_{0}+c_{1}\right)}{r_{0}^{3} c_{0}^{3}} \\
& \times \frac{\frac{(d-1)(d-2)}{4} r_{0}^{4} c_{0}^{2} Z_{0}^{2}-(d-2) \alpha^{2} r_{0}^{2} c_{0} Z_{0} \dot{Z}_{0}+2 \alpha^{4} \dot{Z}_{0}^{2}}{(d-1) Z_{0}-\frac{2 \alpha^{2} \dot{Z}_{0}}{r_{0}^{2} c_{0}}} \\
\mathcal{B}= & \frac{(d-1) c_{0}}{4\left[(d-1) Z_{0}-\frac{2 \alpha^{2} \dot{Z}_{0}}{r_{0}^{2} c_{0}}\right]^{2}}\left[(d-2) r_{0}^{2} c_{0}\left(4 \Lambda+2 V_{0}+Y_{0} a_{t}^{\prime 2}\right)\right. \\
& \left.+2 \alpha^{2}\left(2 \dot{V}_{0}-\dot{Y}_{0} a_{t}^{\prime 2}\right)\right], \\
\mathcal{C}= & \frac{\alpha^{2}}{r_{0}^{2} c_{0}^{2}\left[(d-1) Z_{0}-\frac{2 \alpha^{2} \dot{Z}_{0}}{r_{0}^{2} c_{0}}\right]^{2}}\left\{\alpha^{2} \dot{Z}_{0}\left(4 \Lambda+2 V_{0}-Y_{0} a_{t}^{\prime 2}\right)\right. \\
& -Z_{0}\left[\frac{d-1}{2} r_{0}^{2} c_{0}\left(12 \Lambda+6 V_{0}+Y_{0} a_{t}^{\prime 2}\right)\right. \\
& \left.\left.+\alpha^{2}\left(2 \dot{V}_{0}-\dot{Y}_{0} a_{t}^{\prime 2}\right)\right]\right\} .
\end{aligned}
$$

\section{A. Conductivity reduction induced by charge screening}

Our result for the dc conductivity generalizes those obtained previously from the AdS $R N+$ axions background $(Z=1, Y=1 V=\operatorname{Tr} X)$ [13], and from the backgrounds studied in [21,22] with $Z=1, Y=e^{-\kappa \operatorname{Tr} X} V=\operatorname{Tr} X, \kappa>0$. In these models the Ricci scalar is not coupled directly to the axion. However, the axion-dependent coupling $Y$ has a crucial role in the dc conductivity.

For sufficiently large $\kappa$ and $\alpha$, and for low temperature, the dc conductivity increases with temperature, a behavior previously referred to as "insulating" [21,22]. We note that the physical reason for this behavior is not a smaller scattering time but the simple fact that $Y$ screens the charge 
at low temperature and consequently reduces the conductivity which is proportional to the charge. While the overall temperature dependence is similar to that expected in a system approaching an insulating state, it should be noted that the conductivity is always finite, so the system is metallic in all cases. Moreover, the scattering time, controlled by the parameter $\alpha$, in this model has the same temperature dependence as in the $R N+$ axions background for which no insulating behavior was observed. Very likely, a truly insulating behavior would lead to a qualitative change in the background, something that is not observed in $[21,22]$.

In the following sections we identify a region of parameters in BD holography where we have found similar features which are not induced by charge screening. However, we do not claim that our system is an insulator because the conductivity is never zero, even at zero temperature.

\section{B. The de conductivity in BD axion backgrounds}

In this section we explore the effect of the BD-type coupling $Z$ on the background and on the dc conductivity in $d=3$ boundary dimensions. More specifically, we break translational invariance by using an axion-dependent BD coupling $Z(\operatorname{Tr} X)$. First we consider the simpler case $V=\operatorname{Tr} X=\frac{1}{d-1} \sum_{I} \nabla_{\mu} X^{I} \nabla^{\mu} X^{I}=0$ in Eq. (13) and later study a more general BD-like model where $V=$ $\operatorname{Tr} X \neq 0$ is also present. Initially we restrict our analysis to two space dimensions. The dependence on dimensionality of our results is discussed in the last part of the section.

We note that physically $Z(\operatorname{Tr} X)$ is an axion-dependent gravitational coupling constant that runs from the boundary to the horizon. For that reason we will refer to these axions coupled to the Ricci tensor as gravitational axions. The qualitative effect of this running in the holographic dimension, from weak to strong coupling, is less obvious than for $Y \neq 1$ or in the translationally invariant case.

\section{Momentum relaxation with $Z \neq 1$, $Y=1$ and $V=0$}

We start our analysis with the simpler case of no axion potential and trivial coupling to the Maxwell tensor,

$$
\begin{array}{lll}
Z=e^{\lambda \operatorname{Tr} X}, & \dot{Z}=\lambda Z, & \operatorname{Tr} X=\frac{\alpha^{2}}{r^{2} c}, \\
V=0, & \dot{V}=0, & \\
Y=1, & \dot{Y}=0 . &
\end{array}
$$

Although, a priori, $\lambda$ and $\alpha$ are independent parameters, it is easy to see from Eq. (25) that these parameters appear only in $Z$ as a single parameter $\lambda_{\text {eff }}=\lambda \alpha^{2}$. Translational symmetry is broken for both $\lambda_{\text {eff }}>0$ and $\lambda_{\text {eff }}<0$. In the first case $c\left(r_{0}\right)<1$ while in the second $c\left(r_{0}\right)>1$. However, for $\lambda<0$ the squared "effective mass" $m_{\text {eff }}^{2}$ in Eq. (23) is negative. Therefore, $\lambda_{\text {eff }}$ should be constrained to positive values. ${ }^{8}$

At high temperature, the background tends to AdS $R N+$ axions [13] $(Z=1, Y=1, V=\operatorname{Tr} X)$ : it has a similar blackening factor and $c \rightarrow 1$ for all $r$. The effect of the coupling $Z$ in the background is more evident at low temperatures where $c(r)$ has a stronger dependence on the radial dimension.

Regarding the dc conductivity, this model has qualitatively similar properties to that of $R N+$ axions. For example, for the allowed range of parameters, the dc conductivity increases as the temperature decreases and $\operatorname{Re}\left(\sigma_{\mathrm{dc}}\right)>Y_{0}=1$. Moreover, it is known [13] that for $R N+$ axions in four bulk dimensions, this condition is $\operatorname{Re}\left(\sigma_{\mathrm{dc}}\right)>r_{0}^{d-3}$. In the model Eq. (25) the condition is $\operatorname{Re}\left(\sigma_{\mathrm{dc}}\right)>\left(r_{0}^{2} c_{0}\right)^{\frac{d-3}{2}}$, where $c\left(r_{0}\right)=c_{0}$.

It would be interesting to compare the de conductivity for a fixed scattering time in the model of Eq. (25) with the well-studied AdS $R N+$ axions model. However, it is clear that both models display similar features since the respective actions are related by a conformal transformation. As was explained in detail in Sec. II B 3 for the translationally invariant case, under a conformal transformation, the action of Eq. (13) can be transformed into an action with $Z=1$. The change of the Ricci scalar under this transformation involves additional terms which depend on $\operatorname{Tr} X$ and contain the usual kinetic term proportional to $\sum_{I} \nabla_{\mu} X^{I} \nabla^{\mu} X^{I}, X^{I}=\alpha x^{I}$.

Moreover, as mentioned in Sec. II B 3, in $d=3$ dimensions electromagnetism is conformal. Therefore, the conformal transformation does not change the coupling of the $F^{2}$ term in the action; see Eq. (11). This is consistent with the fact that for the choice of couplings given in Eq. (25), the zero-temperature dc conductivity is larger than 1 , as in the $R N+$ axions model [13]. In higher dimensions, however, it is expected that the model specified by Eq. (25) will yield $\operatorname{Re}\left(\sigma_{\mathrm{dc}}\right)<1$ in some range of parameters. Indeed we will see that this is the case in Sec. IV.

In the next section we study a more general model with a finite potential $(V)$ and nontrivial BD $(Z)$ and Maxwell $(Y)$ couplings in four bulk dimensions.

\section{Momentum relaxation with $Z \neq 1$, $Y \neq 1$ and $V \neq 0$}

Before showing the results for the dc conductivity, it is illuminating to comment on the general features of the gravitational background given in Eq. (14) for the following choice of couplings,

\footnotetext{
${ }^{8}$ As we will demonstrate later in a similar background we have observed that $\lambda<0$ also leads to the violation of the null energy condition. Therefore, we restrict to positive $\lambda_{\text {eff }}$.
} 


$$
\begin{aligned}
& Z=e^{\lambda \operatorname{Tr} X}, \quad \dot{Z}=\lambda Z, \\
& V=\operatorname{Tr} X=\frac{\alpha^{2}}{r^{2} c(r)}, \quad \dot{V}=1, \\
& Y=e^{-\kappa \operatorname{Tr} X}, \quad \dot{Y}=-\kappa Y,
\end{aligned}
$$

where $\alpha, \kappa>0$ and $\lambda$ is real.

The extremal charge density is

$$
\rho_{e}=r_{0}^{2} c_{0} \sqrt{Y(-2 \Lambda-V)} .
$$

It is clear that $\rho_{e}$ decreases as $Y$ decreases, which for the choice of Eq. (26) corresponds to increasing $\alpha$ and $\kappa$. Similarly, for smaller $c\left(r_{0}\right)=c_{0}$ the extremal charge density is smaller.

We now comment on the allowed range of the BDcoupling parameter $\lambda$ according to the properties of the background. Similarly to Sec. III B $1, \lambda>0(Z>1)$ is allowed, in which case the $g / r^{2}$ and $c(r)$ increase monotonically towards the boundary. Contrary to the model of Sec. III B $1, \lambda=0$ is also allowed due to the presence of $V$, which breaks translational invariance. In this case the BD coupling is trivial, $Z=1$, and has been studied previously $[21,22]$.

Moreover, we also find backgrounds satisfying all boundary conditions for $\lambda<0(Z<1)$. However, in this case $g / r^{2}$ does not increase monotonically towards the boundary; there exists a point inside the bulk where the derivative of $g / r^{2}$ vanishes. This suggests the odd feature that the background displays a repulsive behavior between this point and the boundary, which may violate the energy null condition. ${ }^{9}$ In [41] the energy conditions in theories of gravity different from Einstein's gravity have been rederived from Raychaudhury's equation and imposing gravity to be attractive. For the action given in Eq. (13) the null energy condition reduces to

$$
\frac{1}{Z}\left(T_{a b}+\mathcal{Z}_{a b}\right) n^{a} n^{b} \geq 0
$$

for all null vectors $n^{a}$ and where $T_{a b}$ and $\mathcal{Z}_{a b}$ are defined in Eq. (18). We have found that for $\lambda<0$ the background violates the null energy condition Eq. (28). This is easily seen by expressing the background Eq. (14) in the variable $u=r_{0} / r$ and plugging in the null vector $N^{t}=1 / \sqrt{g}$, $N^{r}=\sqrt{g}, N^{i}=0$ in Eq. (28), which reduces to

$$
\begin{aligned}
\left(T_{a b}+\mathcal{Z}_{a b}\right) N^{a} N^{b} & \propto \ddot{Z} \operatorname{Tr} X^{\prime 2}+\dot{Z} \operatorname{Tr} X^{\prime \prime} \\
& \propto c^{\prime}(u)^{2}-2 c(u) c^{\prime \prime}(u) \geq 0 .
\end{aligned}
$$

\footnotetext{
${ }^{9}$ We thank Roberto Emparan for discussion and suggestions on this matter.
}

For $\lambda \geq 0, c^{\prime \prime}(u) \leq 0$ and the null energy condition for this null vector is satisfied; however we have observed that for any $\lambda<0$ this condition is violated.

Regarding the metric function $c(r)$, as the temperature increases, it becomes almost independent of the holographic coordinate $c(r) \approx c\left(r_{0}\right)=c_{0} \rightarrow 1$. The blackening function is also modified in such a way that the geometry approaches that of AdS $R N+$ axions. In the allowed region, $\lambda \geq 0$, the horizon value of $c$ satisfies $c_{0} \leq 1$ and $c_{0}$ decreases for larger $\lambda$. We have already observed this behavior in the model of Sec. III B $1(Y=1$ and $V=0)$. On the other hand, in the forbidden region $\lambda<0, c\left(r_{0}\right)=c_{0}>1$ and $c_{0}$ increases for smaller $\lambda$. We note that at low temperatures the spatial metric functions $g_{i i}=r^{2} c(r)$ could, in principle, be better understood in terms of Lifshitz and hyperscaling violation anomalous exponents, similarly to EMD theories [7]. While we do not rule out that the behavior of $g_{i i}$ close to the horizon may actually be cast using various anomalous exponents, we have not been able to reexpress the metric at low temperatures using a single anomalous exponent.

Finally, in this model, contrary to that of Sec. III B $1, \lambda$ and $\alpha$ are independent parameters. In the presence of $V=\operatorname{Tr} X=\frac{\alpha^{2}}{r_{0}^{2} c_{0}}$, the parameter $\alpha$ appears independently of the parameter $\lambda$ in the action and the equations of motion. Therefore, it is expected that these two cannot be relabeled into a single parameter. For more explicit results regarding the background for different choices of the parameters, see Appendix B.

\section{The de conductivity}

We depict in Figs. 1 and 2 the dc conductivity, Eq. (23), in two space dimensions as a function of temperature for a wide range of the BD parameter $\lambda$ and the charge screening parameter $\kappa$. The effect of $\lambda$ and $\kappa$ is very similar: both control the strength of momentum dissipation.

In Fig. 1 we observe that the increase of either the charge screening or the effective gravitational coupling $(Z>1)$ yields a lower dc conductivity, especially for low temperatures and sufficiently large values of $\lambda>0, \kappa$. We note that in this range of parameters the conductivity is below the bound only because of charge screening.

However, in Fig. 2 we observe that, even though the BD parameter does not appear explicitly in the Maxwell coupling $Y$, its effect is to renormalize the charge screening parameter $\kappa$ through the change in the geometry-more explicitly, through the value of the metric function $c(r)$ at the horizon, $c_{0}$. This is possible even for small $\kappa$. As mentioned before, increasing $\lambda$ leads to a smaller $c_{0}$ and BD coupling which manifests as stronger momentum dissipation. This is a quite interesting and unexpected feature of the model. For instance, the bound in the 

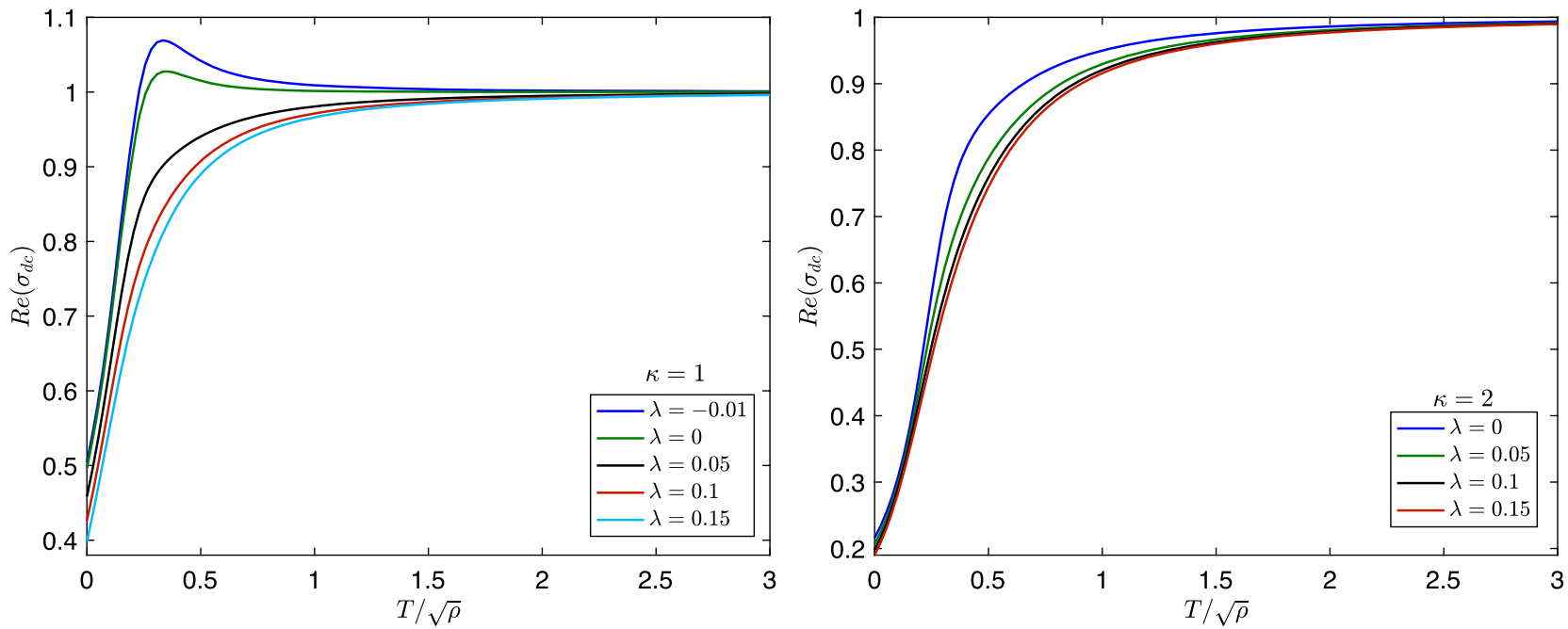

FIG. 1. Temperature dependence of the dc conductivity Eq. (23) for fixed axion parameter $\alpha=1$ and charge density $\rho=1$. The charge screening parameter $\kappa$ and the BD-coupling parameter $\lambda$ are indicated in the plots. The effect of increasing $\lambda$ for fixed $\kappa$ is similar to increasing $\kappa$ for fixed $\lambda$. The case $\lambda<0$ suggests that the effect of a weaker gravitational coupling $Z<1$ on the dc conductivity is to weaken momentum dissipation. However, we stress that this limit violates the null energy condition Eq. (29) and should be excluded.

conductivity of [20] is violated, even for very weak charge screening $\kappa=0.1$, provided that the momentum dissipation by gravitational axions is strong enough $\lambda \geq 0.3$. The change in the temperature dependence of the dc conductivity at low temperature for different values of the parameters is not caused by charge screening but by the effective running of the gravitational coupling. As

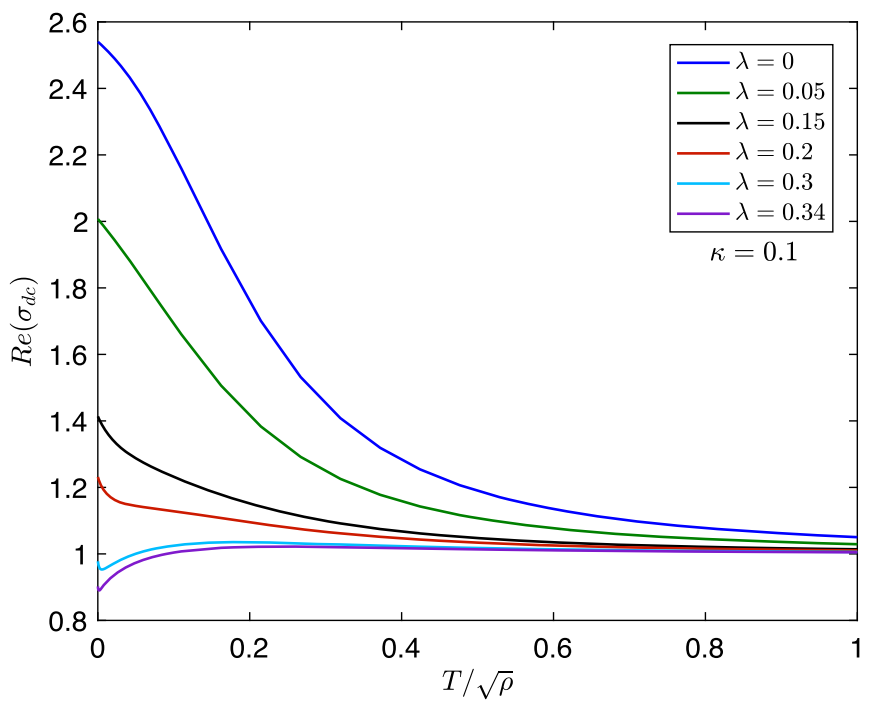

FIG. 2. Temperature dependence of the dc conductivity Eq. (23) for fixed axion parameter $\alpha=1$ and charge density $\rho=1$. The charge screening parameter $\kappa$ and the BD-coupling parameter $\lambda$ are indicated in the plot. The effect of the BD parameter is similar to the charge screening parameter. Increasing $\lambda$ yields a smaller $r_{0}^{2} c_{0}$; as a consequence the first term in Eq. (23), $Y_{0}=\exp \left[-\kappa \alpha^{2} /\left(r_{0}^{2} c_{0}\right)\right]$, decreases. The bound of [20] is violated for large $\lambda$ even for weak charge screening. anticipated in Sec. III A, a decrease in the conductivity for low temperatures sometimes occurs in systems that approach an insulating transition. However, in our case the conductivity, though substantially suppressed, never vanishes. Therefore the model we study is never an insulator.

Notice that in the left plot of Fig. 1 we have included a case in the forbidden range of the BD parameter, $\lambda<0$, which corresponds to a $\mathrm{BD}$ coupling satisfying $Z<1$ and $\dot{Z}=\partial_{\operatorname{Tr} X} Z<0$. We have included this value only to tentatively suggest that the effect of a weaker gravitational interaction could be to effectively reduce the strength of momentum dissipation. Moreover, we have observed that the effective mass in Eq. (23) becomes negative for some $\lambda_{m}<0$, which depends on the rest of the parameters. A negative effective mass has been linked to instabilities of the theory [21]. However, we emphasize that, even for our choice of the BD coupling $Z$ there is a region $\lambda_{m}<\lambda<0$ in which the effective mass is positive but the null energy condition Eq. (29) is violated.

\section{BD HOLOGRAPHY IN HIGHER DIMENSIONS}

So far we have restricted our analysis to $d+1=4$ bulk dimensions. Here we briefly discuss the most salient features of higher dimensional backgrounds. The motivation to study $d>3$ is to observe the explicit effect of the running of the gravitational constant associated to the extra factor $g_{x x}^{\frac{d-3}{2}}=r_{0}^{d-3} c_{0}^{\frac{d-3}{2}}$ in the first term of the conductivity Eq. (23),

$$
\operatorname{Re}\left(\sigma_{\mathrm{dc}}\right)=Y_{0} r_{0}^{d-3} c_{0}^{\frac{d-3}{2}}+\cdots
$$



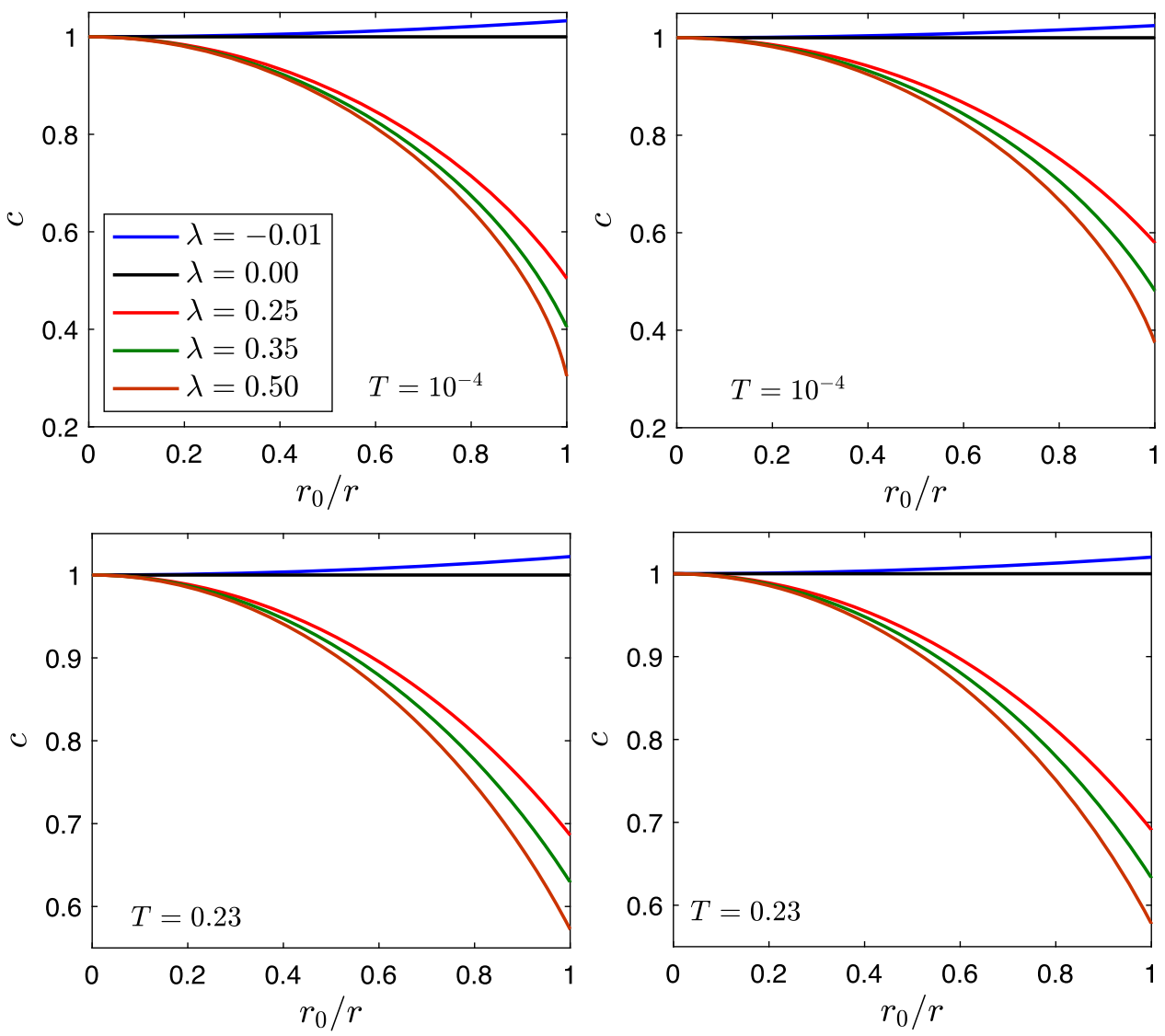

FIG. 3. Metric function $c$, Eq. (14), in $d+1=5$ (left column) and $d+1=6$ (right column) bulk dimensions for the model given in Eq. (26). The temperature is indicated in the plots and the charge density is $\rho=1$. The charge screening parameter $\kappa=1$ and the axion parameter $\alpha=1$. The BD-coupling parameter $\lambda$ is indicated in the legend, which refers to all figures. While the boundary conditions for $\lambda<0$ are satisfied, this case leads to violation of the null energy condition.

The presence of this term is not exclusive to the BD model. Indeed, in EMD models where translational invariance is broken by axion fields, the same factor is present [42]. We note however that, at least in EMD theories where the axions and the dilaton are coupled minimally through a dilaton-dependent coupling constant $[7,12,42]$, the metric function $c$ is trivial, $c=1 .^{10}$ Therefore, in EMD-axion theories, $c_{0}=1$.

We now discuss the two models of Secs. III B 1 and III B 2 for $d=4,5$ boundary spacetime dimensions. In Fig. 3 we plot the metric function $c(r)$ used in the ansatz, Eq. (14), in the model of Eq. (26). In the absence of $V(\operatorname{Tr} X)$, Eq. (25), the background has similar features.

The results, depicted in Fig. 3, indicate that increasing dimensionality decreases the curvature of the metric function $c$. This is more easily seen at low temperature (top row), where $c_{0}=c\left(r=r_{0}\right)$ increases for $\lambda>0$ and decreases for $\lambda<0$. Though not shown in the figure, a similar effect is also observed in the blackening function.

\footnotetext{
${ }^{10} \mathrm{An}$ additional difference in the background is that in the metric ansatz given in Eq. (14), $g_{t t}=-1 / g_{r r}$, which is not the case in an EMD-plus-axion theory $[7,12]$.
}

This is a manifestation of the large-dimensionality limit, $[43,44]$ where the shape of $g$ and $c$ is such that the gravitational effects are stronger closer to the horizon but weaker far from it.

Dimensionality effects on the dc conductivity, Eq. (30), are directly related to the dependence of $c_{0}$ and $r_{0}$ on the dimension. The quantities with tildes are in $\tilde{d}$ dimensions and those without tildes in $d$ dimensions. If $\tilde{d}>d$, we observe that

(i) For $\lambda>0$ and low (high) temperature, $\tilde{c}_{0}>(<) c_{0}$ and $\tilde{r}_{0}<(>) r_{0}$.

(ii) For $\lambda<0$ (forbidden by the null energy condition) and low (high) temperature, $\tilde{c}_{0}<(>) c_{0}$ and $\tilde{r}_{0}>(<) r_{0}$. For the temperature range studied, $\tilde{r}_{0}^{2} \tilde{c}_{0}<r_{0}^{2} c_{0}$. Moreover, for low temperature $r_{0}^{2} c_{0}<1$ but for large temperature $r_{0}^{2} c_{0}>1$. Therefore, for low temperature one expects the suppression of the dc conductivity to be smaller for larger dimensionality.

We show in Fig. 4 that the term $\frac{d-3}{g_{x}}$ in Eq. (30) leads to a suppression of $\operatorname{Re}\left(\sigma_{\mathrm{dc}}\right)$ at low temperature in three space (boundary) dimensions $(d=4)$. In order to isolate the effect of the background we couple the Maxwell field 

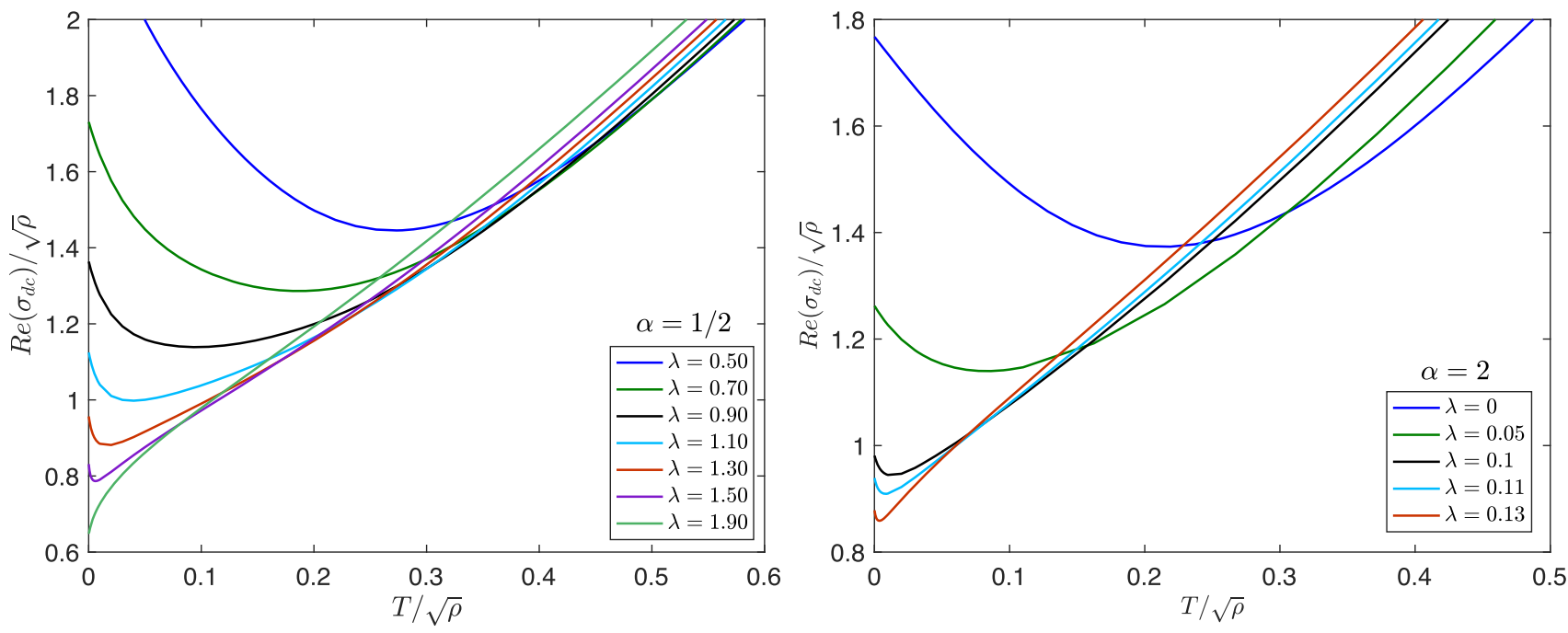

FIG. 4. Zero-frequency conductivity Eq. (23) in $d+1=5$ bulk dimensions in a model with a minimally coupled Maxwell field, Eq. (31). When the BD coupling $\lambda$ is larger than some positive value the dc conductivity at zero temperature is below 1 . This effect is due to the background rather than to an axion-dependent Maxwell coupling as in Fig. 1. The charge density is fixed $\rho=1$.

minimally by setting $Y=1$; namely, the couplings used are those given in Eq. (31):

$$
\begin{aligned}
& Z=e^{\lambda \operatorname{Tr} X}, \quad \dot{Z}=\lambda Z, \\
& V=\operatorname{Tr} X=\frac{\alpha^{2}}{r^{2} c(r)}, \quad \dot{V}=1, \\
& Y=1, \quad \dot{Y}=0 .
\end{aligned}
$$

A similar effect, depicted in Fig. (5), is observed even in the absence of $V(\operatorname{Tr} X)$ in the action (13); namely, we choose the couplings of Eq. (25). As explained in Sec. III B 1 , in the absence of $V$, there is a single parameter that controls the momentum dissipation, $\lambda_{\text {eff }}=\lambda \alpha^{2}>0$. In summary, our results suggest that higher dimension gravitational effects, including those of the gravitational axions, are suppressed except close to the horizon. As a consequence, the conductivity is closer to the $R N$ limit for high temperature. However close to zero temperature, where gravitational effects are still important, the dc conductivity is heavily suppressed for strong momentum relaxation induced by the gravitational axion only. ${ }^{11}$ Therefore, also in this case, the background induces an important suppression of the conductivity without the need for any external source of charge screening. Obviously the system studied in the paper is always a metal; however it would be interesting to explore whether there are other backgrounds within BD holography that reproduce genuine insulating features in the dual field theory.

\footnotetext{
${ }^{11}$ Although, as in the case with charge screening of Sec. III B 2, the conductivity never vanishes.
}

\section{OPTICAL CONDUCTIVITY IN BD HOLOGRAPHY WITH MOMENTUM RELAXATION}

We continue our analysis of transport properties of BD holography by investigating the optical conductivity. We

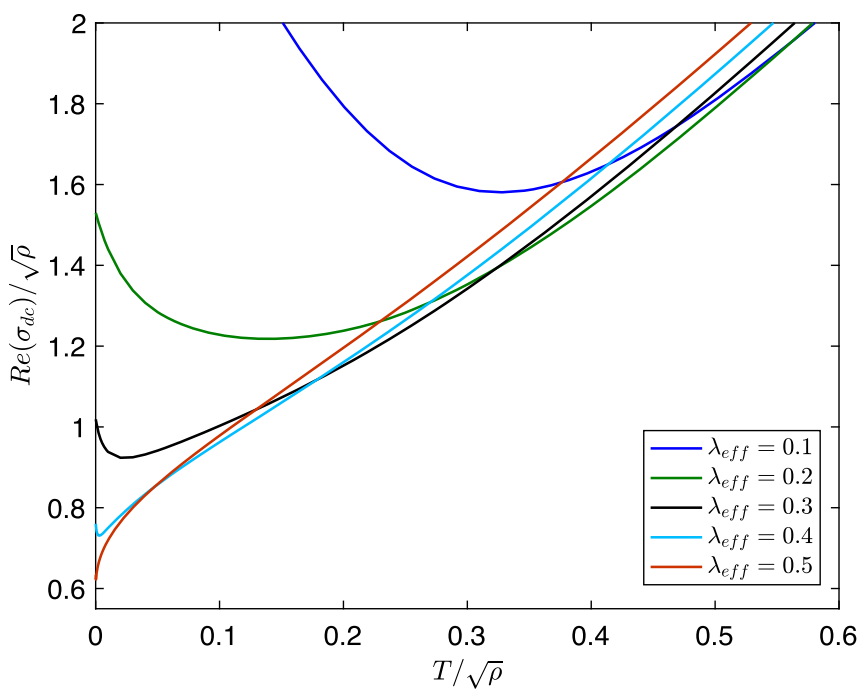

FIG. 5. Zero-frequency conductivity Eq. (23) in $d+1=5$ bulk dimensions in a model with a minimally coupled Maxwell field and $V(\operatorname{Tr} X)=0$, Eq. (25). Similar behavior as in Fig. 4 is observed despite the absence of the usual kinetic term in the action $(V=0)$. Again, the effective change in the gravitational interaction, through the $\mathrm{BD}$ coupling $Z R$, parametrized by $\lambda_{\text {eff }}=\alpha^{2} \lambda$, allows us to decrease the dc conductivity despite the absence of charge screening, $Y=1$. The charge density is fixed, $\rho=1$. 
focus first on the low-frequency scaling of the real part of the conductivity. We found that the conductivity grows linearly with the frequency for low temperatures but strong momentum relaxation. In the second part of the section we show that BD holography, even if combined with other sources of momentum relaxation, does not reproduce the intermediate-frequency scaling of the absolute value and argument of the optical conductivity observed in cuprates.

\section{A. Low-frequency behavior of the conductivity}

In the context of massive gravity the equation for the perturbation leading to the optical conductivity at extremality has been solved analytically for low frequencies by using the method of matched asymptotic expansions [45]. In [13] it was shown that, in the previous model, the dc conductivity is equivalent to that obtained in the $R N+$ axions model, upon a convenient identification of the parameters. Using the method of matched asymptotic expansions we have observed (not shown) that, as in massive gravity, the low-frequency behavior of the optical conductivity of the extremal $R N+$ axions model is also linear in frequency with an always negative slope, which is consistent with Drude physics.

Although we have not been able to obtain analytical results of the low-frequency scaling for arbitrary couplings $Z$ and $Y$, we observe numerically (see Fig. 6) the same linear scaling of the conductivity for small frequencies. Interestingly, provided that momentum relaxation is strong enough, the slope of this linear growth is positive; namely, the conductivity increases with the frequency. This is not exclusive to BD holography; it is also observed for $\lambda=0$ in the limit of strong momentum relaxation induced by the axion coupled to the Maxwell field. This is an interesting feature and we are not aware that it has been reported in holographic systems which do not include charge screening.

At nonzero temperature, the numerical results of Fig. 7 show that the subleading term depends quadratically on the frequency. We conclude therefore that for the general class of models with action Eq. (13), and for low frequency, the optical conductivity is

$$
\operatorname{Re}(\sigma)-\operatorname{Re}\left(\sigma_{\mathrm{dc}}\right)=a \omega+b \omega^{2}+\cdots
$$

where $b \rightarrow 0$ for $T \rightarrow 0$ and, for $T \gg 0$, both constants tend to zero, but $a$ does it faster than $b$. In other words, at large temperature we have observed a subleading contribution dominated by $\omega^{2}$ while in the limit of zero temperature it is proportional to $\omega^{1}$. As mentioned above, for $Z=1$ and a minimally a coupled Maxwell field $(Y=1)$, the constants $a$ and $b$ are always negative, describing the broadening of the Drude peak. For $Y \neq 1$ and both $Z \neq 1$ and $Z=1, a$ is negative when $\operatorname{Re}\left(\sigma_{\mathrm{dc}}\right)>1$ and positive when $\operatorname{Re}\left(\sigma_{\mathrm{dc}}\right)<1$.

For $Y \neq 1$ and both at zero (not shown) and nonzero temperature (bottom left plot in Fig. 7), we have observed a range of parameters for which the optical conductivity has a local maximum for relatively small frequencies. In Fig. 7 we observe that in the high temperature limit the local peak is smeared. A similar feature in the de conductivity has been recently reported in [21]. Similarly to the phenomenology observed in the temperature dependence of the dc conductivity, we believe that, in the range of parameters of Fig. 7, this intermediate peak is a consequence of
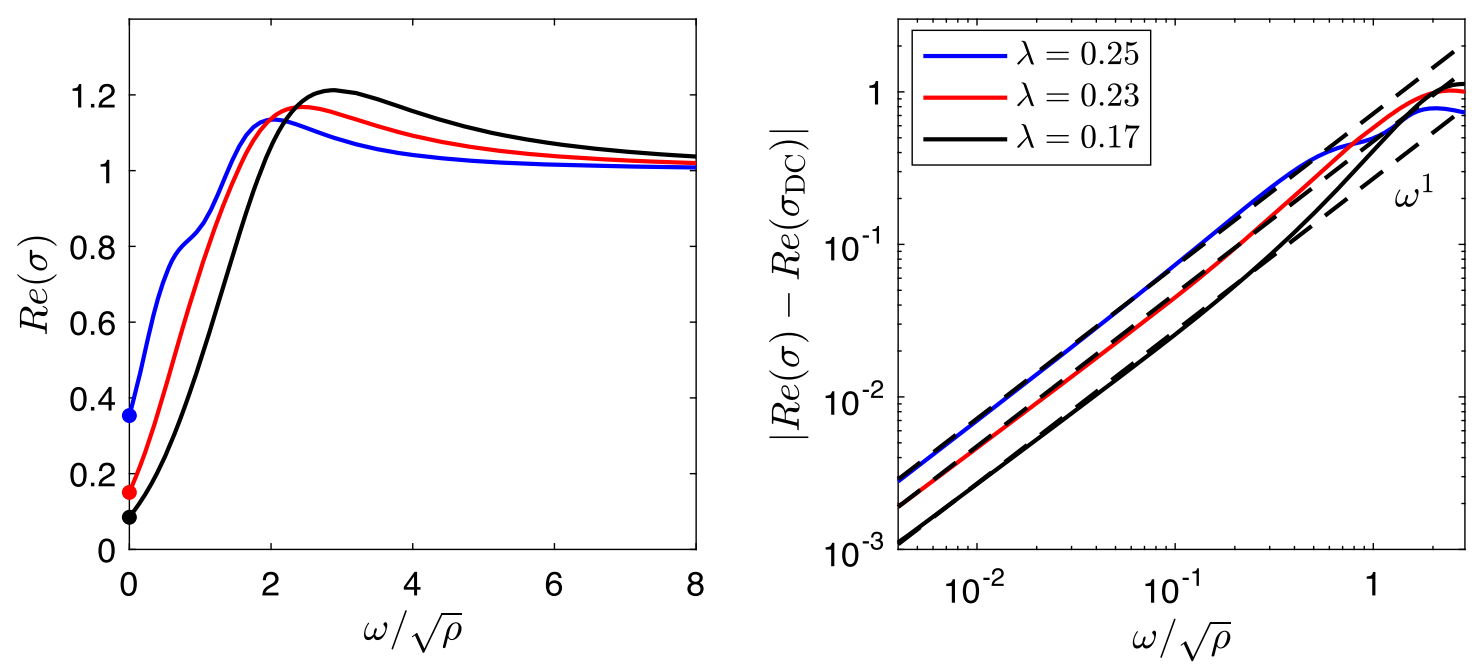

FIG. 6. Zero-temperature [extremal background of Eqs. (13) and (26)] optical conductivity for strong breaking of translational symmetry. The charge screening parameter is $\kappa=1$ and the axion parameter is $\alpha=1$ (blue lines), $\alpha=1.5$ (red lines) and $\alpha=2$ (black continuous lines). The BD-coupling parameter $\lambda$, given in the legend, is fixed close to the maximum value allowed by the background boundary conditions. The extremal charge density is $\rho=1$. The dashed black lines correspond to the linear scaling $\beta \omega$, where $\beta$ is fixed from the lowest frequency point of the numerical data. 

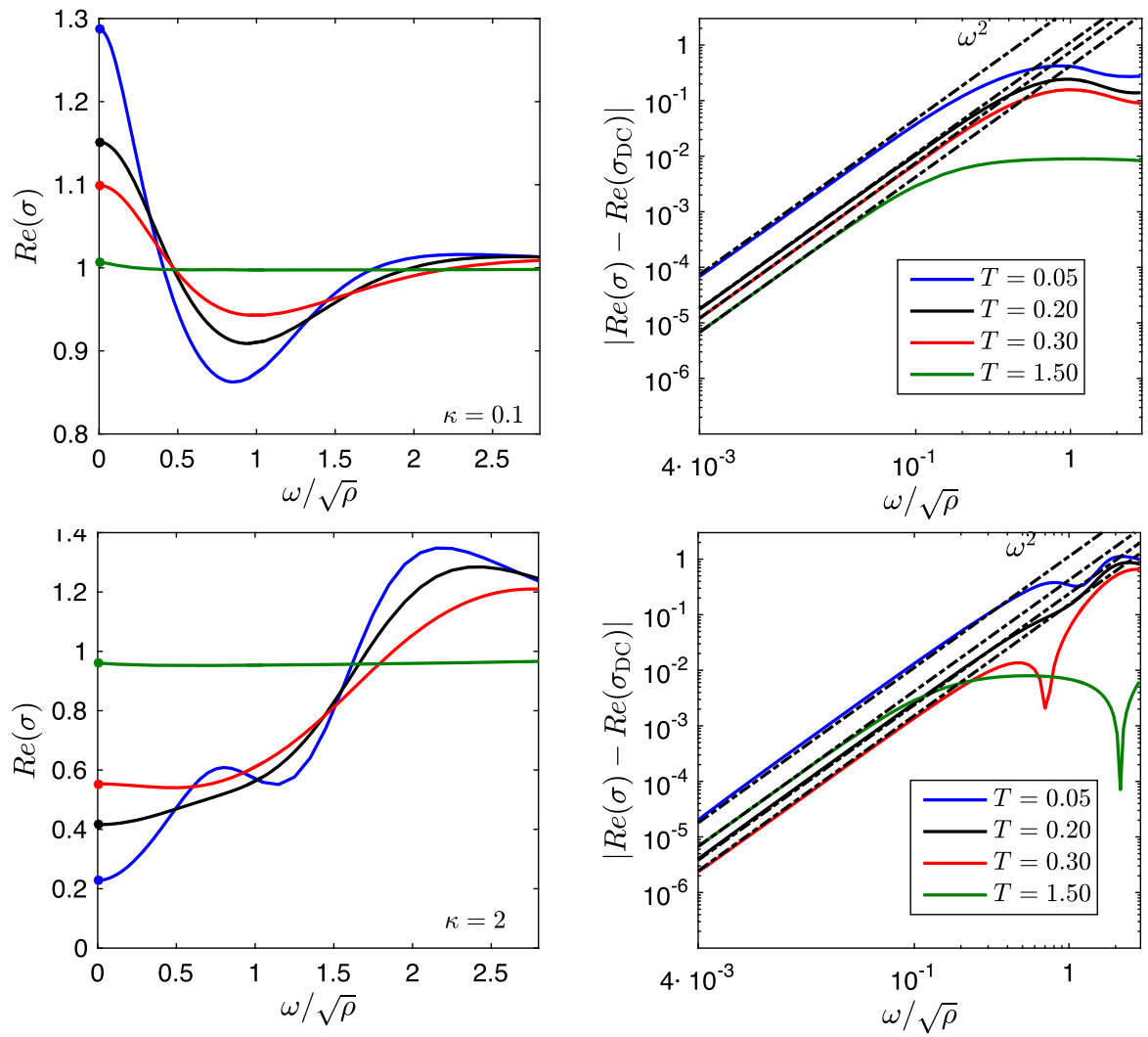

FIG. 7. Optical conductivity at finite temperature in the background given in Eqs. (13) and (26). Each line corresponds to a fixed temperature, indicated in the legend of the right-hand plots. The dashed-dotted black lines correspond to the quadratic scaling and are fixed in the same way as in Fig. 6. As temperature decreases (blue lines) a slight disagreement is observed. We expect that for near extremal solutions the frequency scaling is given by Eq. (32). The charge density $\rho=1, \alpha=1, \lambda=0.15$ and $\kappa$ is indicated in the lefthand plots.

charge screening induced by a nontrivial axion-dependent Maxwell coupling and therefore it is not a precursor of insulating behavior. Indeed the conductivity is always finite.

We have observed that a stronger gravitational interaction (larger BD coupling $Z$ ) has a similar impact on the intermediate peak with increasing momentum dissipation. As was mentioned in Sec. III B 2, in $d=3$ boundary dimensions this is a nontrivial effect: a conformal transformation of the action Eq. (13) with $d>3$ renormalizes the Maxwell coupling in an analogous way to the conformal transformation of the BD model; see Eq. (11). However, for $d=3$ the Maxwell coupling is invariant under such transformation and one could expect therefore the Maxwell coupling to be unchanged by a change in the BD coupling. Nonetheless, from a holographic point of view, it is known that the observables in the boundary theory are roughly determined by the gravitational background (plus boundary values of bulk fields). Therefore, at intermediate energy scales, and despite the fact that the Maxwell coupling is invariant under a conformal transformation for $d=3$, one should expect the features of the BD background at intermediate length scales to determine the optical conductivity.

\section{B. Argument and modulus of the optical conductivity in BD gravity with momentum relaxation}

A well-known property of the optical conductivity in most cuprates is that for intermediate frequencies the module of the conductivity scales as $\omega^{-2 / 3}$. It has been recently claimed [24] that a holographic setup where momentum relaxation is introduced by a modulating chemical potential shares similar properties. However, we note that this holographic setup does not reproduce another property of the optical conductivity in cuprates: the argument of the conductivity is constant in the same range of frequencies. In this section we study whether field theory duals of gravity models with different channels of momentum dissipation can reproduce these features of the optical conductivity in cuprates.

Results for different values of the parameters are depicted in Fig. 8.

Either the constant argument or the desired $2 / 3$ powerlaw decay can be observed for some values of the parameters. However, it is clear from our results that even by fine-tuning all the available parameters we could not reproduce both features for a single set of parameters. 

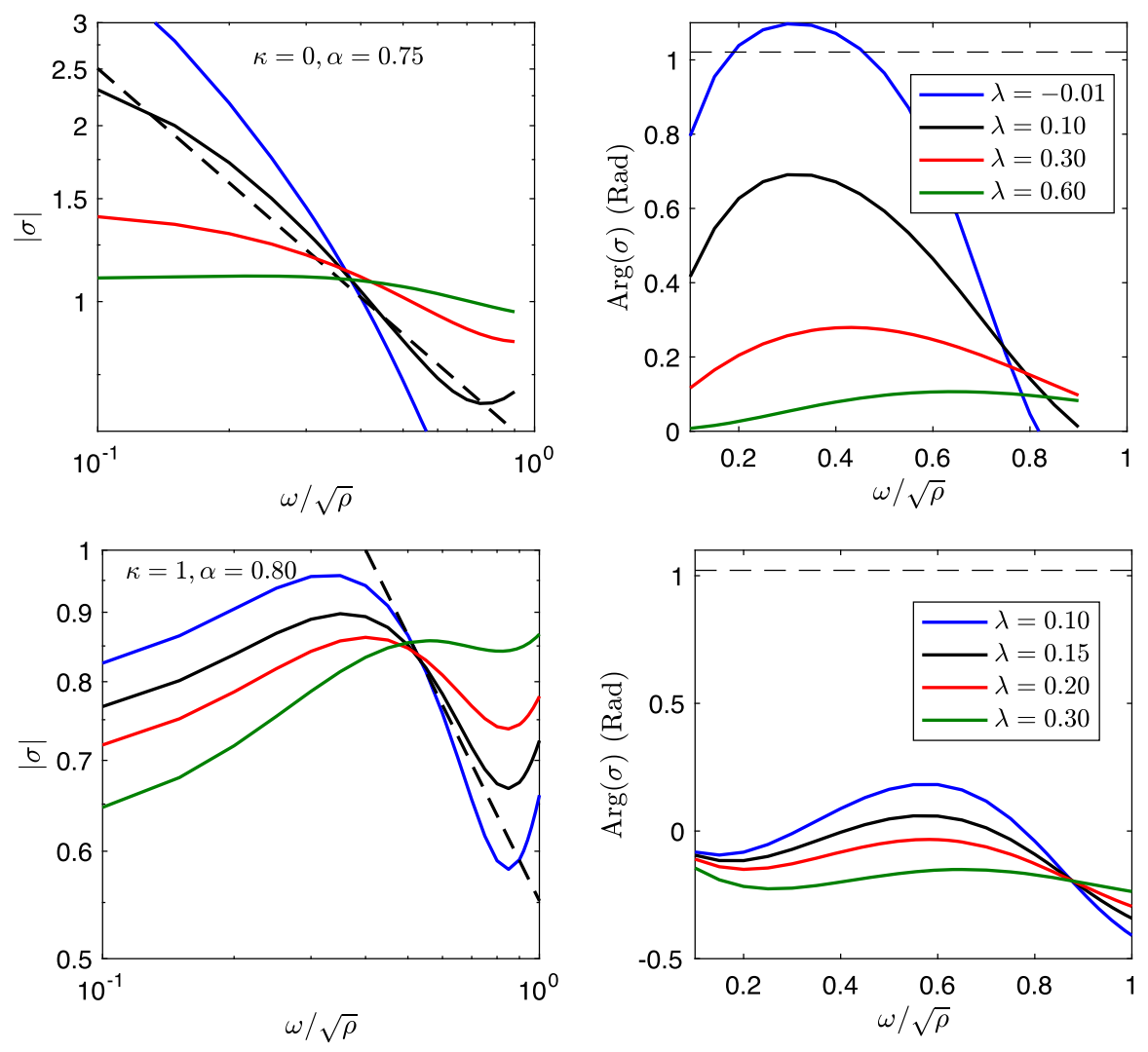

FIG. 8. Absolute value and argument of the optical conductivity for different values of the parameters. The temperature and charge density are $T=0.05$ and $\rho=1$. The BD model with the couplings of Eq. (26) does not describe the experimental behavior for intermediate frequencies observed for cuprates. The dashed lines are the exponent $\xi,|\sigma|=\omega^{a}, a=1.35-2=-0.65$, and $\arg (\sigma)=$ $\frac{\pi}{2}(2-1.35)=1.01 \mathrm{Rad}$, according to experiments.

\section{RATIO OF SHEAR VISCOSITY AND ENTROPY DENSITY IN BD WITH MOMENTUM RELAXATION}

In this section we study the ratio $\eta / s$ between the shear viscosity $\eta$ and the density of entropy $s$ for BD holography with momentum relaxation. In a quantum field theory the viscosity is defined through the Kubo formula:

$$
\eta=-\lim _{\omega \rightarrow 0} \frac{1}{\omega} \operatorname{Im} G_{T^{x y}}^{R} T^{x y}(\omega, q=0),
$$

where $T^{x y}$ is the $x y$ component of the stress-energy tensor. In order to compute the viscosity in the model of Eq. (13) we use the membrane paradigm in a similar way as in Sec. II B for the calculation of the regular part of the de conductivity. While we restrict ourselves to numerical results we expect that, as shown in [46], it should be possible to derive quasianalytical approximations at low and large temperatures. ${ }^{12}$

\footnotetext{
${ }^{12}$ An analytical calculation in terms of the background expansion close to the boundary is possible; however, the background needs to be computed numerically.
}

Previously it has been reported that in the presence of momentum relaxation [46-50] or anisotropy $[51-53]^{13}$ the ratio is temperature dependent and in most cases below the KSS bound. We find similar results in the case of BD holography. For our analysis we use the couplings of Eq. (26) with $V=\operatorname{Tr} X$ (Fig. 9) and $V=0$ (Fig. 10) which include, as a particular case, some of the previously studied cases of AdS $R N+$ axions [46,50]. In the full range of parameters we have explored, the ratio decreases with temperature and is always below the KSS bound. It also decreases as the strength of momentum relaxation increases, by any of the channels explored. It seems that it can be made arbitrarily close to zero even for a finite momentum relaxation. We do not have a clear understanding of the physical reasons behind this behavior; however we note that similar results have been observed [55] in the context of the quark-gluon plasma with

\footnotetext{
${ }^{13}$ We note that in these models the effective mass of the graviton is nonzero. As shown in [54] this is a necessary condition to observe violation of the KSS bound. However, in theories without translational invariance and massless gravitons [54] the KSS bound remains valid. Our model falls in the first class of theories.
} 


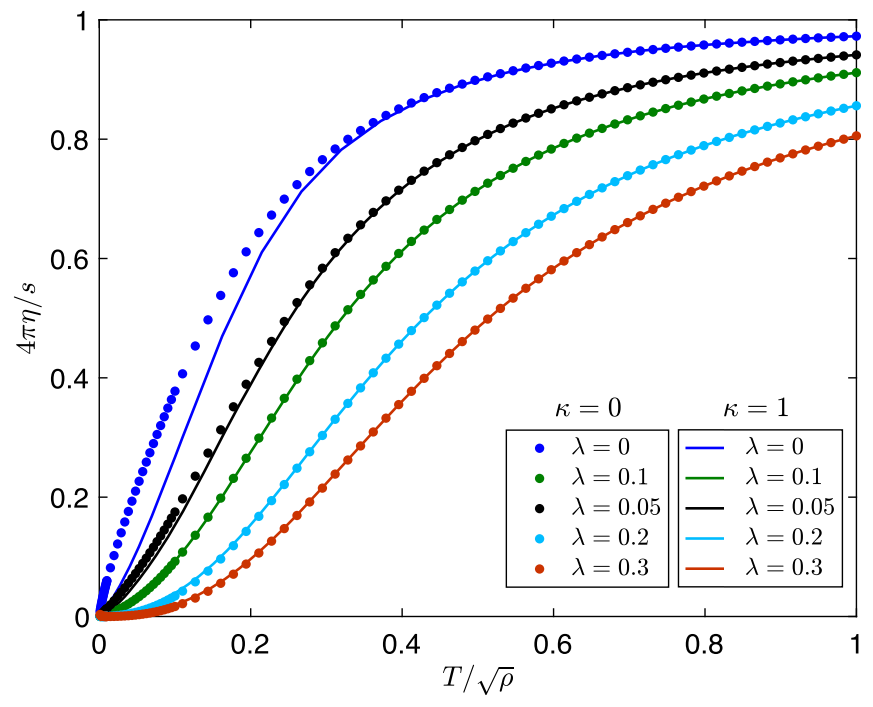

FIG. 9. Shear viscosity to entropy density ratio $(\eta / s)$ for the couplings of Eq. (26) in the model defined in Eqs. (13) and (26). The axion parameter $\alpha=1$ and the charge density $\rho=1$.

quenched impurities in the limit at which the phenomenon of Anderson localization becomes important.

\section{OUTLOOK AND CONCLUSIONS}

The potential interest of BD backgrounds in holography is well beyond the problems discussed in the paper. For instance, the entanglement entropy depends explicitly on the gravity coupling constant and it is also very sensitive to the strength of bulk interactions. Therefore we expect that

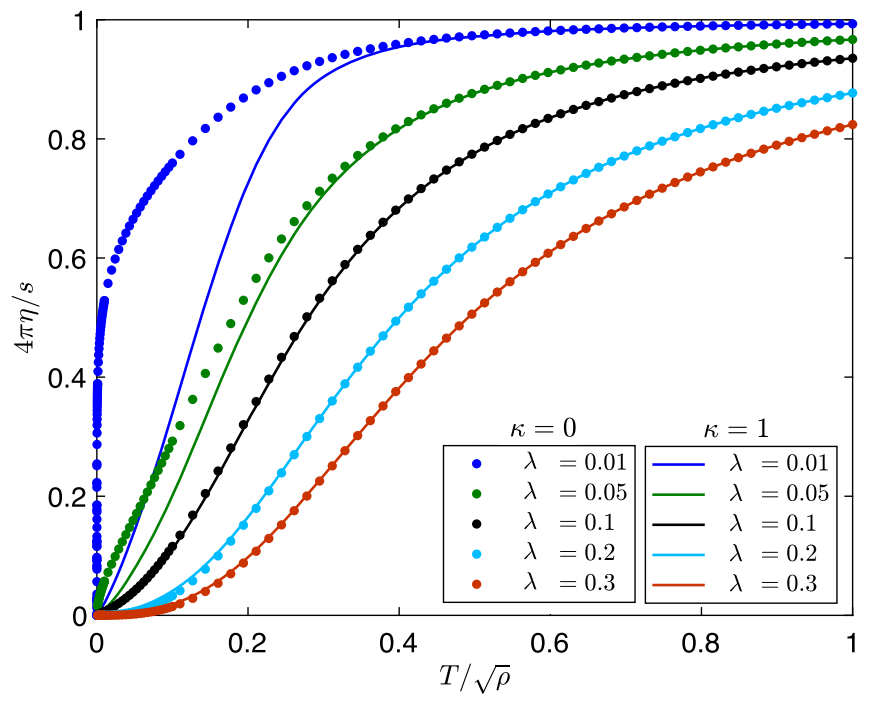

FIG. 10. Shear viscosity to entropy density ratio $(\eta / s)$ for the couplings of Eq. (26) in the model defined in Eqs. (13) and (26) but taking $V=0$ and $\dot{V}=0$. The axion parameter $\alpha=1$ and the charge density $\rho=1$. As discussed in Sec. III B 1 , for $\kappa=0 \Rightarrow$ $Y=1$ (dots) the theory has a single parameter that controls momentum dissipation, namely $\lambda_{\text {eff }}=\lambda \alpha^{2}$. the holographic entanglement entropy in inhomogeneous BD backgrounds may reveal interesting features not found in previous holographic duals. These features would not only be present to leading order (area of minimal surface) but also in the quantum correction originated by the entanglement between the bulk and the minimal surface [56]. Another topic of potential interest is that of holographic superconductivity. It is well known that the width of the coherence peak as well as the ratio of the zerotemperature order parameter to the critical temperature are useful indicators of the strength of the interactions binding the condensate. For the former, values much larger than the Bardeen-Cooper-Schrieffer prediction, suggesting strong interactions, are expected. It would be interesting to investigate whether it is possible to tune this ratio in $\mathrm{BD}$ backgrounds. That would be a smoking gun that the scalar in BD backgrounds effectively controls the interactions in the bulk. Finally, we note that the introduction of randomness in the scalar is qualitatively different from other forms of disorder used in holography. It amounts to a random strength of the gravitational interaction. Mobile charge introduced through the gauge field will feel these random interactions not very differently from the way in which electrons feel quench impurities. This is in stark contrast with the effect of a random chemical potential, quite popular in holography, where the mobile carriers are by construction randomly but homogeneously distributed through the sample. Coherence phenomena like Mott-Anderson localization could not be observed in this setting. We plan to address some of these problems in the near future.

In summary, we have investigated the transport properties of strongly coupled field theories whose gravity dual is a Brans-Dicke action where gravity is mediated by both a tensor, the graviton, and a scalar that depends on the radial dimension. In the translationally invariant limit we have computed analytically several transport properties. The finite part of the dc conductivity $\sigma_{Q}$, expressed in terms of thermodynamic quantities, is different from the universal prediction for EMD backgrounds [10]; however the shear viscosity ratio is still given by the KSS bound. Similar results apply to other generalized $\mathrm{f}(\mathrm{R})$ gravity backgrounds that can be mapped onto BD. The difference with EMD models is that the entropy does not hold an area law as it also depends on the value of the scalar at the horizon.

Momentum relaxation is induced by a gravitational axion, namely, the linear coupling of the Ricci tensor and the axion. Following the procedure pioneered by Donos and Gauntlett [40] we compute analytically the dc conductivity as a function of the metric at the horizon which is evaluated numerically. In $d+1=4$ bulk dimensions momentum relaxation by $\mathrm{BD}$ axions is qualitatively similar to the results obtained by other mechanisms of momentum relaxation $[13,21,22]$ in the limit of strong charge screening. Interestingly for strongly coupled gravitational axions that induce strong momentum relaxation, the conductivity bound 
[20] is violated for any finite charge screening induced by the electromagnetic axion [21,22]. In higher spatial dimensions the dc conductivity for sufficiently strong momentum relaxation decreases in the low temperature limit. This suggests that the analogous conductivity bound is violated even if there is no coupling between the axion and the Maxwell field. We have also computed numerically the optical conductivity in BD backgrounds with momentum relaxation. For sufficiently strong breaking of translational invariance, the conductivity grows linearly with the frequency in the limit of small frequencies and very low temperatures though it remains finite for any temperature and frequency. We have also evaluated numerically the modulus and the argument of the optical conductivity for different momentum relaxation channels in order to find out whether the phenomenology of this model is similar to that of the cuprates for intermediate frequencies. Our results are not very encouraging. For any value of the parameters we could not reproduce the experimental results for both quantities simultaneously. Finally, we have shown that the shear viscosity to entropy ratio decreases with temperature and the KSS bound is violated by any strength of the momentum relaxation.

\section{ACKNOWLEDGMENTS}

A. R. B. thanks Roberto Emparan for illuminating discussions. We also thank Matteo Baggioli and Oriol Pujolàs for useful comments on the manuscript. A. M. G. acknowledges support from EPSRC, Grant No. EP/I004637/1. B. L. is supported by CAPES/COT Grant No. 11469/13-17. A. R. B. acknowledges support from the Department of Physics and the Theory of Condensed Matter group of the University of Cambridge as well as the Cambridge Philosophical Society.

\section{APPENDIX A: AN ASYMPTOTICALLY ADS BRANS-DICKE BLACK HOLE}

As we have previously discussed in Sec. II B 3, the action (1) can be brought to the Einstein frame via a conformal transformation. In this frame, the BD action maps to an EMD model. Solutions for this action have been widely studied for different choices of potentials [5-7,29]. For completeness, we give here a particular explicit black brane solution with AdS asymptotics. In the language of Ref. [7] this corresponds to a $\delta=\gamma$ solution:

$$
\begin{array}{r}
\mathrm{d} \bar{s}^{2}=-f(r) \mathrm{d} t^{2}+\frac{\mathrm{d} r^{2}}{f(r)}+r^{2} R(r) \delta_{i j} \mathrm{~d} x^{i} \mathrm{~d} x^{j} \\
f(r)=\frac{2 \Lambda\left(\alpha^{2}+1\right)^{2} b^{2 \gamma}}{(d-1)\left(\alpha^{2}-d\right)} r^{2(1-\gamma)}-\frac{m}{r^{(d-1)(1-\gamma)-1}} \\
+\frac{2 q^{2}\left(\alpha^{2}+1\right)^{2} b^{-2(d-2) \gamma}}{(d-1)\left(\alpha^{2}+d-2\right)} r^{2(d-2)(\gamma-1)}
\end{array}
$$

$$
\begin{gathered}
R(r)=\left(\frac{b}{r}\right)^{2 \gamma} \\
\bar{\phi}(r)=\frac{(d-1) \alpha}{2\left(1+\alpha^{2}\right)} \log \frac{b}{r} \\
\bar{V}(\bar{\phi})=2 \Lambda e^{\frac{4 \alpha \bar{\phi}}{d-1}}=\left(\frac{b}{r}\right)^{2 \gamma} \\
\bar{a}_{t}{ }^{\prime}=-\frac{q Y}{r^{d-1}}\left(\frac{b}{r}\right)^{-(d-3) \gamma}
\end{gathered}
$$

where we defined $\gamma=\alpha^{2} /\left(1+\alpha^{2}\right)$, with $\alpha$ as in Eq. (11). This solution has four free parameters $(\gamma, b, q, m) \cdot \gamma$ is a function of the Brans-Dicke parameter $\xi$ :

$$
\begin{aligned}
\gamma & =\frac{\alpha^{2}}{1+\alpha^{2}} \\
& =\frac{1}{1+\alpha^{-2}} \\
& =\frac{(d-3)^{2}}{(d-1)^{2}+8+4(d-1) \xi} .
\end{aligned}
$$

The black brane horizon radius is found by imposing $f\left(r_{0}\right)=0$. This allows us to solve for one of the parameters as functions of $r_{0}$ and the others, e.g. $m=m\left(r_{0}, q, b, \gamma\right)$. The parameter $b$ sets the scale of the dilaton $\bar{\phi}$ and can be set to unity by a coordinate rescaling.

An interesting case is given by $d=3$, where $\gamma=\alpha=0$ and the scalar field is constant. In this case the solution above reduces to the well-known AdS Reissner-Nordström solution. Note that $\gamma=\alpha=0$ also for $\xi \rightarrow \infty$, which is the well-known Einstein limit of Brans-Dicke theory. In this limit, the solution also reduces to $d+1$ dimensional Reissner-Nordström. $q$ is the charge density of the background and $m$ is related to the energy density [ArnowittDeser-Misner (ADM) mass] as we will discuss below.

To construct an asymptotically AdS black hole solution for our Brans-Dicke theory (1) we take the inverse conformal mapping on the solution above. The BransDicke black hole is then given by

$$
\begin{aligned}
\mathrm{d} s^{2} & =-A(r) \mathrm{d} t^{2}+B(r) \mathrm{d} r^{2}+r^{2} c(r) \delta_{i j} \mathrm{~d} x^{i} \mathrm{~d} x^{j} \\
A(r) & =\phi^{-\frac{2}{d-1}} f(r) B(r)=\frac{\phi^{-\frac{2}{d-1}}}{f(r)}, \\
c(r) & =\phi^{-\frac{2}{d-1}} R(r)=\left(\frac{b}{r}\right)^{\frac{2(d-5)}{d-3} \gamma} \\
V(\phi) & =2 \Lambda \phi^{2} \\
a_{t}^{\prime} & =-\frac{q Y}{r^{d-1}}\left(\frac{b}{r}\right)^{-(d-3) \gamma} \\
\phi(r) & =\left(\frac{b}{r}\right)^{\frac{2(d-1) r}{d-3}} .
\end{aligned}
$$


Note that in particular this transformation preserves the position of the horizon $r_{0}$. The temperature can then be computed,

$$
\begin{aligned}
4 \pi T= & \left|A\left(r_{0}\right)\right| \\
= & \frac{\left(d-\alpha^{2}\right) m}{\alpha^{2}+1} r_{0}^{(d-1)(\gamma-1)} \\
& -\frac{4 q^{2}\left(\alpha^{2}+1\right) b^{-2(d-2) \gamma}}{\alpha^{2}+d-2} r_{0}^{(2 d-3)(\gamma-1)-\gamma},
\end{aligned}
$$

which again reduces to the $R N$ temperature for $\gamma=\alpha=0$. The free energy density can be computed by the properly renormalized Euclidean action. We refer the curious reader to [57] for the details of the calculation and just quote the answer here,

$$
\begin{aligned}
f= & \beta\left(\frac{(d-1) b^{(d-1) \gamma} m}{\alpha^{2}+1}\right)-\frac{b^{(d-1) \gamma} r_{0}^{(d-1)(1-\gamma)}}{4 \pi} \\
& -\beta \frac{q^{2}}{2((d-3)(1-\gamma)+1)} \frac{1}{r_{0}^{1+(d-3)(1-\gamma)}} \\
= & \beta \epsilon-s-\beta \mu q
\end{aligned}
$$

for $\beta=T^{-1}$ and we defined
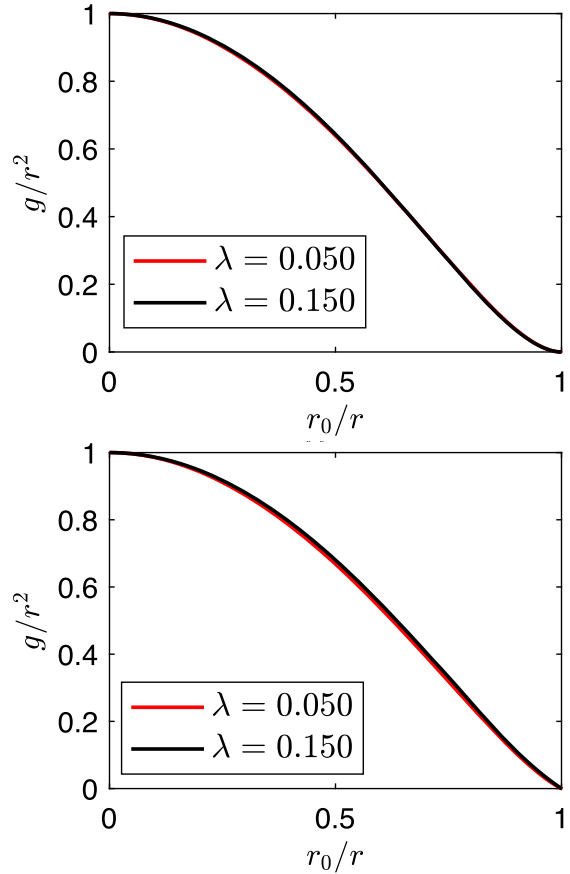

$$
\begin{aligned}
\epsilon & =\frac{(d-1) b^{(d-1) \gamma} m}{\alpha^{2}+1}, \\
s & =\frac{b^{(d-1) \gamma} r_{0}^{(d-1)(1-\gamma)}}{4 \pi}, \\
\mu & =\frac{q^{2}}{2((d-3)(1-\gamma)+1)} \frac{1}{r_{0}^{1+(d-3)(1-\gamma)}},
\end{aligned}
$$

which are the energy density (ADM mass), entropy density and chemical potential, respectively. In particular note that the above satisfy the first law $\mathrm{d} \epsilon=T \mathrm{~d} s+\mu \mathrm{d} \rho$ for $4 \pi \rho=\int_{\mathcal{M}} \star F=q$, the charge density.

\section{APPENDIX B: GRAVITATIONAL BACKGROUND WITH $Z \neq 1, Y=1$ AND $V \neq 1$ IN FOUR BULK DIMENSIONS}

In Fig. 11 we show the metric functions, defined in Eq. (14), at nonzero temperature for the model defined in Eq. (13) and couplings given in Eq. (25).

The lines correspond to a fixed $\lambda_{\text {eff }} \equiv \lambda \alpha^{2}$ but different $\lambda$ and $\alpha$, defined in Eq. (26). Most notably, the function $c$ at the horizon shows a difference of about $20 \%$, while the blackening factor is very similar throughout the bulk. Figure 11 shows that the model defined in Eqs. (13) and (26) contains three independent parameters, $\kappa, \alpha$ and $\lambda$.
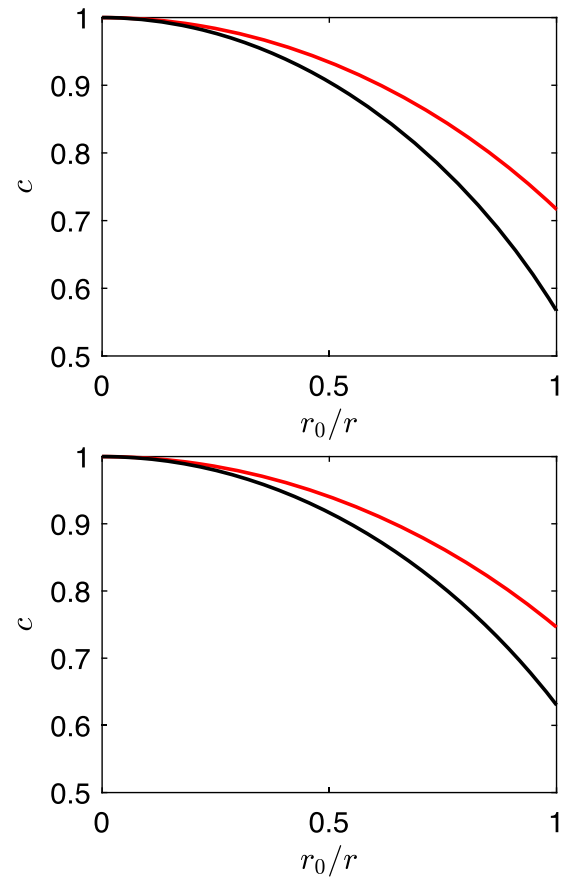

FIG. 11. Metric functions $g$ (blackening factor) and $c$, Eq. (14), for two different temperatures: (top row) $T=10^{-4}$ and (bottom row) $T=0.08$. We fix the charge density $\rho=1, \kappa=1$ and $\lambda_{\text {eff }}=\lambda \alpha^{2}=0.15$. Each line corresponds to $\alpha^{2}=\lambda_{\text {eff }} / \lambda$ for the corresponding $\lambda$, which is given in the legends. The legends also refer to the right-hand figures. Moreover, for a fixed temperature and $\lambda_{\text {eff }}$, the dc conductivity is different for the two choices of $\lambda$ and $\alpha$. We conclude that, in the presence of $V$ in the action, $\lambda$ and $\alpha$ are two independent parameters associated with the translational symmetry breaking. 
[1] H. Goenner, Some remarks on the genesis of scalar-tensor theories, Gen. Relativ. Gravit. 44, 2077 (2012).

[2] C. Brans and R. H. Dicke, Mach's principle and a relativistic theory of gravitation, Phys. Rev. 124, 925 (1961).

[3] G. Kang, Black hole area in Brans-Dicke theory, Phys. Rev. D 54, 7483 (1996).

[4] A. Sheykhi and M. M. Yazdanpanah, Thermodynamics of charged Brans-Dicke AdS black holes, Phys. Lett. B 679, 311 (2009).

[5] A. Sheykhi, M. H. Dehghani, and S. H. Hendi, Thermodynamic instability of charged dilaton black holes in AdS spaces, Phys. Rev. D 81, 084040 (2010).

[6] C. Charmousis, B. Goutéraux, B. S. Kim, E. Kiritsis, and R. Meyer, Effective holographic theories for low-temperature condensed matter systems, J. High Energy Phys. 11 (2010) 151.

[7] E. Kiritsis and J. Ren, On holographic insulators and supersolids, J. High Energy Phys. 09 (2015) 168.

[8] G. Policastro, D. T. Son, and A. O. Starinets, Shear Viscosity of Strongly Coupled $N=4$ Supersymmetric Yang-Mills Plasma, Phys. Rev. Lett. 87, 081601 (2001).

[9] S. K. Chakrabarti, S. Chakrabortty, and S. Jain, Proof of universality of electrical conductivity at finite chemical potential, J. High Energy Phys. 02 (2011) 073.

[10] S. Jain, Universal thermal and electrical conductivity from holography, J. High Energy Phys. 11 (2010) 092.

[11] R. A. Davison, B. Goutéraux, and S. A. Hartnoll, Incoherent transport in clean quantum critical metals, J. High Energy Phys. 10 (2015) 112.

[12] A. M. García-García and A. Romero-Bermúdez, Drude weight and Mazur-Suzuki bounds in holography, Phys. Rev. D 93, 066015 (2016).

[13] T. Andrade and B. Withers, A simple holographic model of momentum relaxation, J. High Energy Phys. 05 (2014) 101.

[14] A. Adams and S. Yaida, Disordered holographic systems: Functional renormalization, Phys. Rev. D 92, 126008 (2015).

[15] D. Areán, A. Farahi, L. A. Pando Zayas, I. S. Landea, and A. Scardicchio, Holographic superconductor with disorder, Phys. Rev. D 89, 106003 (2014).

[16] S. A. Hartnoll and J.E. Santos, Disordered Horizons: Holography of Randomly Disordered Fixed Points, Phys. Rev. Lett. 112, 231601 (2014).

[17] D. K. O'Keeffe and A. W. Peet, Perturbatively charged holographic disorder, Phys. Rev. D 92, 046004 (2015).

[18] A. M. García-García and B. Loureiro, Marginal and irrelevant disorder in Einstein-Maxwell backgrounds, Phys. Rev. D 93, 065025 (2016).

[19] M. Araujo, D. Arean, and J. M. Lizana, Noisy branes, J. High Energy Phys. 07 (2016) 091.

[20] S. Grozdanov, A. Lucas, S. Sachdev, and K. Schalm, Absence of Disorder-Driven Metal-Insulator Transitions in Simple Holographic Models, Phys. Rev. Lett. 115, 221601 (2015).

[21] M. Baggioli and O. Pujolas, On holographic disorder-driven metal-insulator transitions, arXiv:1601.07897.

[22] B. Goutéraux, E. Kiritsis, and W.-J. Li, Effective holographic theories of momentum relaxation and violation of conductivity bound, J. High Energy Phys. 04 (2016) 122.
[23] S. Gopalakrishnan, M. Müller, V. Khemani, M. Knap, E. Demler, and D. A. Huse, Low-frequency conductivity in many-body localized systems, Phys. Rev. B 92, 104202 (2015).

[24] G. T. Horowitz and J. E. Santos, General relativity and the cuprates, J. High Energy Phys. 06 (2013) 087.

[25] G. W. Gibbons and K.-i. Maeda, Black holes and membranes in higher-dimensional theories with dilaton fields, Nucl. Phys. B298, 741 (1988).

[26] D. Garfinkle, G. T. Horowitz, and A. Strominger, Charged black holes in string theory, Phys. Rev. D 43, 3140 (1991).

[27] B. Goutéraux and E. Kiritsis, Generalized holographic quantum criticality at finite density, J. High Energy Phys. 12 (2011) 036.

[28] C. H. Brans, The roots of scalar-tensor theory: An approximate history, arXiv:gr-qc/0506063.

[29] C. J. Gao and S. N. Zhang, Higher-dimensional dilaton black holes with cosmological constant, Phys. Lett. B 605, 185 (2005).

[30] I. L. Shapiro and H. Takata, Conformal transformation in gravity, Phys. Lett. B 361, 31 (1995).

[31] I. L. Shapiro, On the conformal transformation and duality in gravity, Classical Quantum Gravity 14, 391 (1997).

[32] T. P. Sotiriou and V. Faraoni, $f(R)$ theories of gravity, Rev. Mod. Phys. 82, 451 (2010).

[33] T. P. Sotiriou, S. Liberati, and V. Faraoni, Theory of gravitation theories: A no-progress report, Int. J. Mod. Phys. D 17, 399 (2008).

[34] I. L. Shapiro and H. Takata, One-loop renormalization of the four-dimensional theory for quantum dilaton gravity, Phys. Rev. D 52, 2162 (1995).

[35] R. Brustein and A. J. M. Medved, Ratio of shear viscosity to entropy density in generalized theories of gravity, Phys. Rev. D 79, 021901 (2009).

[36] R. Brustein, D. Gorbonos, and M. Hadad, Wald's entropy is equal to a quarter of the horizon area in units of the effective gravitational coupling, Phys. Rev. D 79, 044025 (2009).

[37] S. Nojiri and S. D. Odintsov, Non-singular modified gravity unifying inflation with late-time acceleration and universality of viscous ratio bound in $\mathrm{F}(\mathrm{R})$ theory, Prog. Theor. Phys. Suppl. 190, 155 (2011).

[38] S. Chatterjee, M. Parikh, and S. Sarkar, The black hole membrane paradigm in $f(R)$ gravity, Classical Quantum Gravity 29, 035014 (2012).

[39] R. Pourhasan, Spacetime entanglement with $f(R)$ gravity, J. High Energy Phys. 06 (2014) 4.

[40] A. Donos and J. P. Gauntlett, Thermoelectric DC conductivities from black hole horizons, J. High Energy Phys. 11 (2014) 081.

[41] J. Santos, J. S. Alcaniz, M. J. Reboucas, and F. C. Carvalho, Energy conditions in $f(R)$-gravity, Phys. Rev. D 76, 083513 (2007).

[42] B. Goutéraux, Charge transport in holography with momentum dissipation, J. High Energy Phys. 04 (2014) 181.

[43] R. Emparan, D. Grumiller, and K. Tanabe, Large- $D$ Gravity and Low- $D$ Strings, Phys. Rev. Lett. 110, 251102 (2013).

[44] A. M. García-García and A. Romero-Bermúdez, Conductivity and entanglement entropy of high dimensional holographic superconductors, J. High Energy Phys. 09 (2015) 033. 
[45] R. A. Davison, Momentum relaxation in holographic massive gravity, Phys. Rev. D 88, 086003 (2013).

[46] S. A. Hartnoll, D. M. Ramirez, and J. E. Santos, Entropy production, viscosity bounds and bumpy black holes, J. High Energy Phys. 03 (2016) 170.

[47] X. H. Ge, Y. Ling, C. Niu, and S. J. Sin, Thermoelectric conductivities, shear viscosity, and stability in an anisotropic linear axion model, Phys. Rev. D 92, 106005 (2015).

[48] M. Sadeghi and S. Parvizi, Hydrodynamics of a black brane in Gauss-Bonnet massive gravity, Classical Quantum Gravity 33, 035005 (2016).

[49] P. Burikham and N. Poovuttikul, Shear viscosity in holography and effective theory of transport without translational symmetry, arXiv:1601.04624.

[50] Y. Ling, Z.-Y. Xian, and Z. Zhou, Holographic shear viscosity in hyperscaling violating theories without translational invariance, arXiv:1605.03879.

[51] A. Rebhan and D. Steineder, Violation of the Holographic Viscosity Bound in a Strongly Coupled Anisotropic Plasma, Phys. Rev. Lett. 108, 021601 (2012).
[52] K. A. Mamo, Holographic RG flow of the shear viscosity to entropy density ratio in strongly coupled anisotropic plasma, J. High Energy Phys. 10 (2012) 070.

[53] S. I. Finazzo, R. Critelli, R. Rougemont, and J. Noronha, Momentum transport in strongly coupled anisotropic plasmas in the presence of strong magnetic fields, Phys. Rev. D 94, 054020 (2016).

[54] L. Alberte, M. Baggioli, and O. Pujolas, Viscosity bound violation in holographic solids and the viscoelastic response, J. High Energy Phys. 07 (2016) 074.

[55] I. Giannakis, D. Hou, J.-R. Li, and H.-c. Ren, Low shear viscosity due to Anderson localization, Phys. Rev. D 77, 027701 (2008).

[56] T. Faulkner, A. Lewkowycz, and J. Maldacena, Quantum corrections to holographic entanglement entropy, J. High Energy Phys. 11 (2013) 074.

[57] A. Sheykhi and H. Alavirad, Topological black holes in Brans-Dicke-Maxwell theory, Int. J. Mod. Phys. D 18, 1773 (2009). 